\title{
Loss Mechanism of Static Interstage Components of Multistage Centrifugal Compressors for Integrated Blade Design
}

\author{
Chunjun Ji, ${ }^{1,2}$ Chunyang Li $\mathbb{D}^{1},{ }^{1}$ Junyi Fang, ${ }^{1}$ and Qi Sun ${ }^{3}{ }^{3}$ \\ ${ }^{1}$ School of Energy and Power Engineering, Dalian University of Technology, Dalian 116024, China \\ ${ }^{2}$ Key Laboratory of Ocean Energy Utilization and Energy Conservation of Ministry of Education, Dalian University of Technology, \\ Dalian 116024, China \\ ${ }^{3}$ Institute of Engineering Thermophysics, Chinese Academy of Sciences, Beijing 100190, China
}

Correspondence should be addressed to Chunyang Li; 906549286@qq.com

Received 9 August 2018; Accepted 25 November 2018; Published 5 December 2018

Academic Editor: Eusebio Valero

Copyright (C) 2018 Chunjun Ji et al. This is an open access article distributed under the Creative Commons Attribution License, which permits unrestricted use, distribution, and reproduction in any medium, provided the original work is properly cited.

\begin{abstract}
Although centrifugal compressors are widely used in construction, they consume a large amount of energy; in existing multistage centrifugal compressors, there is a serious pressure loss of $\sim 15.13 \%$ when gas flows through the diffuser, bend, and return channel. In this study, we analyze the loss mechanisms of these stages in detail, using computational fluid dynamics. Based on this analysis, we present a new type of integrated blade, connecting the diffuser, bend, and return channels, which can eliminate the airflow stall phenomenon. Through effective control of the airflow spreading process, we minimized losses in the component, which improved its efficiency by $4.39 \%$ and increased the pressure ratio by $2.86 \%$ relative to a compressor without the newly-designed integrated blade. The concepts used in the creation of this component can provide a reference for the future design of blades for flow through parts of multistage compressors.
\end{abstract}

\section{Introduction}

The continued development of computing technology has made its combination with mathematical processes the conventional method for solving practical engineering problems. Computational fluid dynamics (CFD) is such a discipline where this combination is applied to engineering solutions. Using basic physical laws and discretization methods, mathematical models of complex objects can be created. The differential equations associated with these models are subsequently solved using appropriate computing algorithms, to obtain the spatial and temporal distribution of the desired physical properties of a fluid. Thus, CFD techniques can reduce the cost and duration of experiments. As a result of these advantages, CFD techniques have become an important scientific research method.

Centrifugal compressors are a class of machinery universally used in industry [1] that have a wide range of applications and consume a large amount of energy. The demands of industry, resulting from the need to sustain and accelerate economic growth, have meant that multistage centrifugal compressors are widely used. As large amounts of energy loss are induced in their diffuser, bend, and return sections, many scholars have carried out extensive and detailed research on these three regions, to improve the efficiency of a complete machine. For instance, Filipenco et al. [2] and Bonaiuti et al. [3] found that diffusers with vanes can improve the performance of compressors. Zhao et al. [4] revealed the energy loss mechanism of diffuser blades, by comparing the performance of different diffusers in centrifugal compressors with large flow coefficients; they observed that the change in the circumferential flow angle at the diffuser inlet affects the inlet incidence of the diffuser vane and the vane loading distribution [5]. This information is useful for enhancing compressor performance. Hildebrandt and Schilling [6] performed aerodynamic characterization of two and three-dimensional return blades using numerical and experimental methods and pointed the loss mechanisms of return blades, for similar reasons.

Impeller-diffuser interaction has also been studied for improving the performance of centrifugal compressors, as it is an important part of the complete machine [7]. Tamaki [8] 
performed experiments using seven different vane diffusers and the same impeller, to study the influence of diffuser blade installation angle on the performance of a centrifugal compressor. Ubaldi et al. [9] analyzed the effect of vaned diffusers on impeller flow. Hosseini et al. noted that centrifugal compressors possess an optimal radial clearance ratio that maximizes the pressure recovery coefficient and stage efficiency and minimizes the loss coefficient. Shum et al. [10] independently observed an optimal radial gap ratio, noting decreases in slips and blockages and increases in loss when this parameter was increased. Gallier [11] studied excitation mechanisms for impeller-diffuser interaction in a centrifugal compressor. Robinson et al. [12] reported the occurrence of mechanical excitation with the impeller when this component was too close to the diffuser.

Although scholars have focused on the research and design of individual components, airflow between different stages of multistage centrifugal compressors has not been considered as a integrated. The object of this paper is to improve the intermediate stage of a vertical section multistage centrifugal compressor that has total losses of $15.13 \%$ when gas flows through the diffuser, the bend, and the return. Using the results from the literature cited above and analysis of the loss mechanisms of existing compressor models performed using CFD, we propose a new type of integrated blade, to connect the diffuser, the bend, and the return channel. This new blade improves the uniformity of the gas flow angle in the bend, effectively controlling the airflow spreading process and reducing flow loss. Hence, the efficiency and pressure ratio of the improved compressor containing this integrated blade increased by $4.39 \%$ and $2.86 \%$, relative to a standard model.

\section{Mathematical Model}

2.1. Geometric Parameters of the Original Model. In this paper we investigated the intermediate stage of a vertical split type multistage centrifugal compressor with 13 rear curved blades. The geometric parameters and operating conditions of this component are summarized in Table 1 . The structure of the meridional section of the compressor is illustrated in Figure 1, while Figure 2 depicts a solid model of the studied object.

\subsection{Calculation and Analysis of Original Model Flow Field.}

To define a mathematical model for the intermediate section of the compressor, we established a computation domain consisting of an inlet channel, an impeller, a vaneless diffuser, and the bend, return, and outlet flow channels. The efficiency of the entire stage was calculated to be $76.97 \%$. The efficiency of gas flow through the impeller was $92.1 \%$, indicating that $15.13 \%$ of the flow loss can be attributed to the static components of the diffuser, the bend, and the return channels. To aid in analysis of the flow loss mechanism of the intermediate compressor stage, we have included maps of pressure, airflow angle, and velocity distribution.

In addition to a drop in total pressure, a dense isoline distribution can be observed when gas flows from the diffuser channel to the return channel, using the pressure map of the
TABLE 1: Geometric parameters and operating conditions of the original model.

\begin{tabular}{lc}
\hline Parameter & Value \\
\hline Number of impeller blades, $\mathrm{Z}_{1}$ & 13 \\
Speed, $\mathrm{N}(\mathrm{rpm})$ & 8129 \\
Average impeller inlet diameter, $\mathrm{D}_{1}(\mathrm{~mm})$ & 326.54 \\
Impeller outlet diameter, $\mathrm{D}_{2} /$ outlet width, $\mathrm{b}_{2}(\mathrm{~mm})$ & $600 / 39$ \\
Number of diffuser blades, $\mathrm{Z}_{2}$ & 0 \\
Diffuser inlet diameter, $\mathrm{D}_{3} /$ inlet width, $\mathrm{b}_{3}(\mathrm{~mm})$ & $660 / 39$ \\
Diffuser outlet diameter, $\mathrm{D}_{4} /$ outlet width, $\mathrm{b}_{4}(\mathrm{~mm})$ & $960 / 39$ \\
Bend inner arc radius, $\mathrm{R}_{1} /$ outer arc radius, $\mathrm{R}_{2}(\mathrm{~mm})$ & $18 / 57$ \\
Number of return vanes, $\mathrm{Z}_{3}$ & 10 \\
Return inlet diameter, $\mathrm{D}_{5} /$ inlet width, $\mathrm{b}_{5}(\mathrm{~mm})$ & $946 / 32$ \\
Average return outlet diameter, $\mathrm{D}_{6} /$ outlet width, $\mathrm{b}_{6}$ & $466 / 52.63$ \\
(mm) & Air \\
Medium & 7.6 \\
Design condition mass flow, $\mathrm{Q},(\mathrm{kg} / \mathrm{s})$ & $76.97 \%$ \\
Efficiency, $\eta$ & 1.432 \\
Pressure ratio, $\varepsilon$ &
\end{tabular}

meridional section illustrated in Figure 3. In particular, there is a noticeable low pressure region in the main flow area of the return channel, at the boundary between the diffuser and bend channels, as seen in Figure 4. In contrast, from Figure 5, we note that the contours representing the static pressure distribution in the diffuser channel are relatively uniform, indicating that the suffusion of gas in the main flow region of this component is fairly even.

Figure 6 shows the flow angle distribution in the meridional plane, which indicates that the overall flow direction in the diffuser channel is fairly consistent, changing only after gas passes through the bend. This behavior differs from the air flow angle observed in the return channel, where the flow is already disordered. Figure 7 shows a plot of the variation of the air flow angle in each section of the bend as a function of the distance from the hub to the shroud. Gas turns in the bend because of centrifugal force, and the direction of airflow changes. Although the angle of airflow in the inlet section of the bend remains relatively constant, gas transport in the middle section is no longer uniform, and the fluid flow is separated. This separation is compounded when the gas interacts with the return blade, resulting in a more disordered flow.

Figure 8 shows the distribution of velocity vectors in the original compressor model, with the scale increased by an additional 50\%. From Figure 9, it can be seen that the gas begins to separate at the inlet to the suction surface of the return blade, resulting in a small range of return motion, to the downstream of the return blade, which accumulates a stronger vortex, resulting in large energy loss.

To further analyze the reduction in airflow efficiency through each of the diffuser, the bend, and the return channel, 


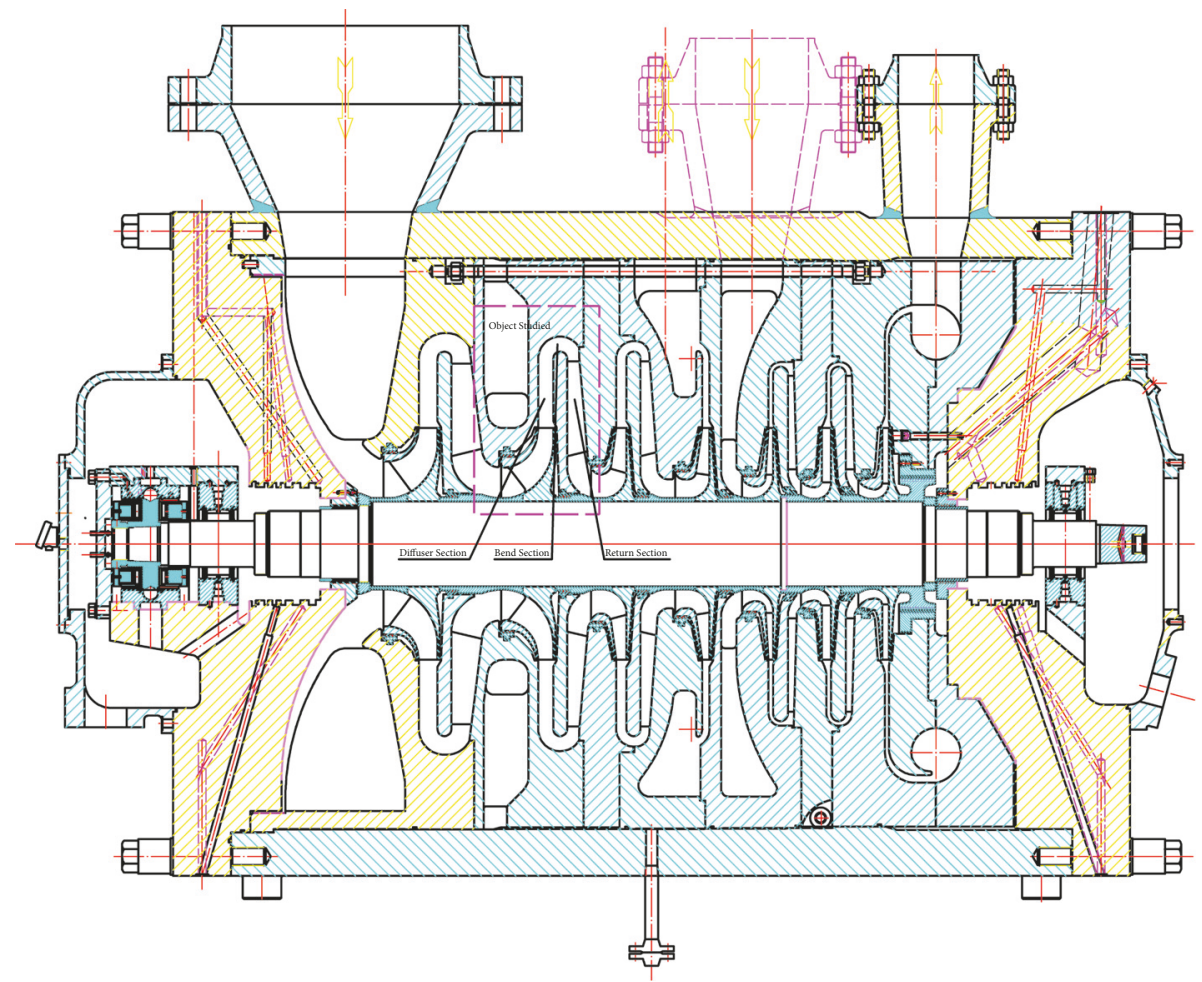

FIGURE 1: Meridional section of a centrifugal compressor.

we defined the total pressure loss coefficient, $\xi$, and the static pressure recovery coefficient, $\mathrm{C}_{\mathrm{P}}[2]$, as follows:

$$
\begin{aligned}
\xi & =\frac{\left(P_{\text {in }}-P_{L}\right)}{(1 / 2) \rho_{\text {in }} c_{i n}{ }^{2}}, \\
C p & =\frac{P_{s x}-P_{s}}{P_{t}-P_{s}},
\end{aligned}
$$

where in (1), $P_{i n}, \rho_{i n}$, and $c_{i n}$ are the mean total pressure, average density, and average absolute velocity of the airflow on the computational domain inlet cross section and $P_{L}$ is the average value of the total pressure in the calculated points of the selected section. In (2), $P_{s}$ and $P_{s x}$ are the mean static pressure of the diffuser inlet section and the selected cross section (the diffuser outlet section in this paper), respectively, and $P_{t}$ is the average value of the total pressure in the diffuser inlet section. A summary of these parameters, for each section of the original compressor model, is included in Table 2.

From Table 2, it can be seen that the maximum total pressure loss is observed in the return channel, which accounts for $44.48 \%$ of the total pressure loss from static components. Losses in the diffuser and the bend account for $30.69 \%$ and $24.83 \%$ of the total pressure loss from static components, respectively.

Analysis of gas flow in the diffuser, bend, and return channels of the original compressor model elucidates a range of conclusions. We note that total pressure loss is concentrated primarily at the boundary layer of the diffuser. In this section, this loss is due to friction caused by the
TABLE 2: Total pressure loss and static pressure recovery coefficients for each section of the original compressor model.

\begin{tabular}{lc}
\hline Section & Value \\
\hline Total pressure of diffuser inlet cross section $(\mathrm{Pa})$ & 153,566 \\
Static pressure of diffuser inlet cross section $(\mathrm{Pa})$ & 134,344 \\
Total pressure of diffuser outlet cross section $(\mathrm{Pa})$ & 151,259 \\
Static pressure of diffuser outlet cross section $(\mathrm{Pa})$ & 143,559 \\
Total pressure loss coefficient of diffuser & 0.089 \\
Static pressure recovery coefficient of diffuser & 0.479 \\
Total pressure of exit section of bend $(\mathrm{Pa})$ & 149,401 \\
Total pressure loss coefficient of bend & 0.072 \\
Total pressure of outlet section of return $(\mathrm{Pa})$ & 146,059 \\
Total pressure loss coefficient of return & 0.129 \\
\hline
\end{tabular}

viscosity of the fluid. In spite of this loss, the static pressure rise of the gas in the diffuser is good. Pressure loss in the bend is concentrated between the hub and the shroud. This is because of the centrifugal force applied to make the gas turn around the bend, which consumes energy and makes the gas flow direction uneven. In addition to this, the effect of wall friction, dictated by the viscosity of the gas, also contributes to pressure loss in this region. Finally, the largest loss of pressure is observed in the return section, which is primarily due to the uneven direction of gas exiting the bend. The interaction of this uneven flow with the return blade causes gas separation, explaining the large loss in pressure in this section. 


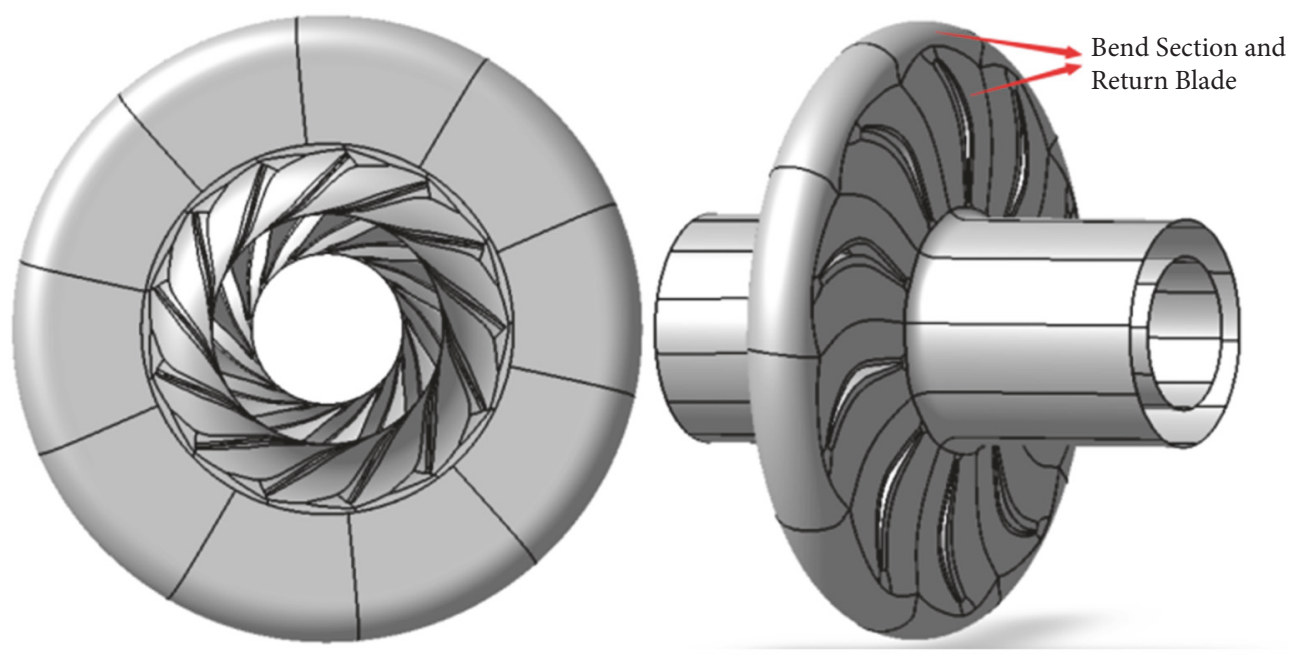

FIGURE 2: 3D model of the original compressor used in analysis.

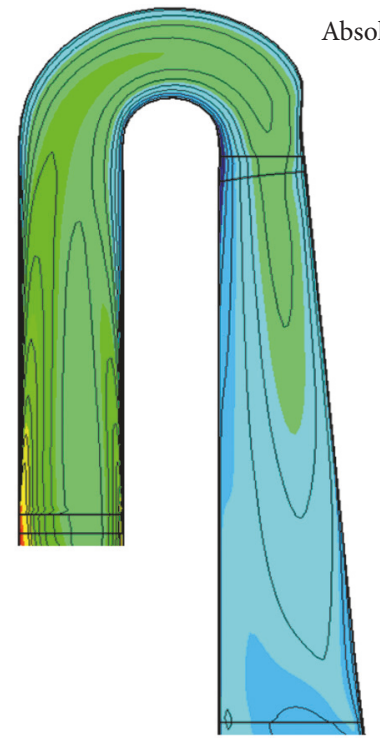

Absolute Total Pressure $(\mathrm{Pa})$

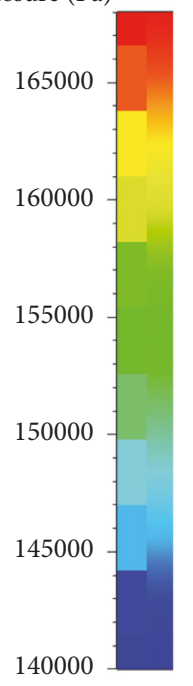

FIGURE 3: Total pressure distribution in the meridional section of the original compressor model.

2.3. Design and Modeling of Integrated Blade. Based on the conclusions made in the previous section, we have concentrated on developing an integrated blade to improve gas flow through the static elements of the diffuser, the bend, and the return units, to reduce flow loss in a compressor. Blade design is constrained primarily by its meridional profile and inlet design angle in the compressor. The second of these parameters is analyzed in further detail in Section 4.

(1) Meridional Profile of the Integrated Blade. Figure 10 depicts a schematic of the blade profiles in the original and improved compressor models, to illustrate the modifications made in designing the integrated blade. To reduce gas flow loss from the impeller blade outlet to the stationary parts of the compressor and improve its efficiency, the transition section

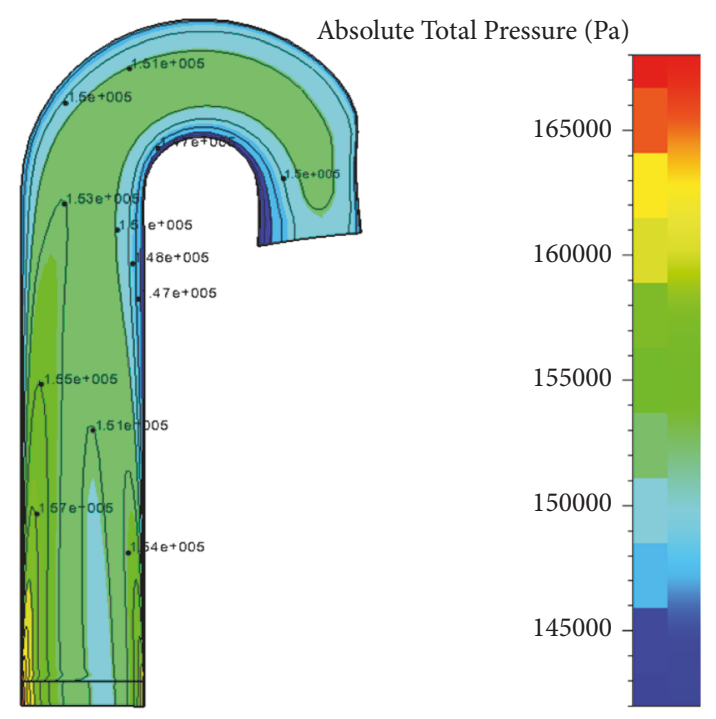

FIGURE 4: Total pressure distribution in the diffuser of the original compressor model.

was designed as a convergent channel. Hence, the ratio of the inlet width of the diffuser to the outlet width of the impeller $\left(b_{3} / b_{2}\right)$ was set to 0.8 . Subsequent expansion sections were designed to be of equal width; i.e., the inlet and outlet widths of the diffuser were made equal $\left(b_{4}=b_{3}\right)$. The inner arc radius of the bend remained the same at $18 \mathrm{~mm}$, while the hub side profile of the return channel was made tangent to the inner arc radius of the bend; i.e., circular arc 12 is tangent to straight line 23 at point 2 .

To keep the axial length of the model unchanged, the side profile of the shroud of the return channel was inclined to the hub; i.e., straight line 45 is inclined to straight line $45^{\prime}$, and the outer arc of the bend was made tangent to point 5 ' and point 6 ', to define the shroud cover profile of the return and diffuser channels. 


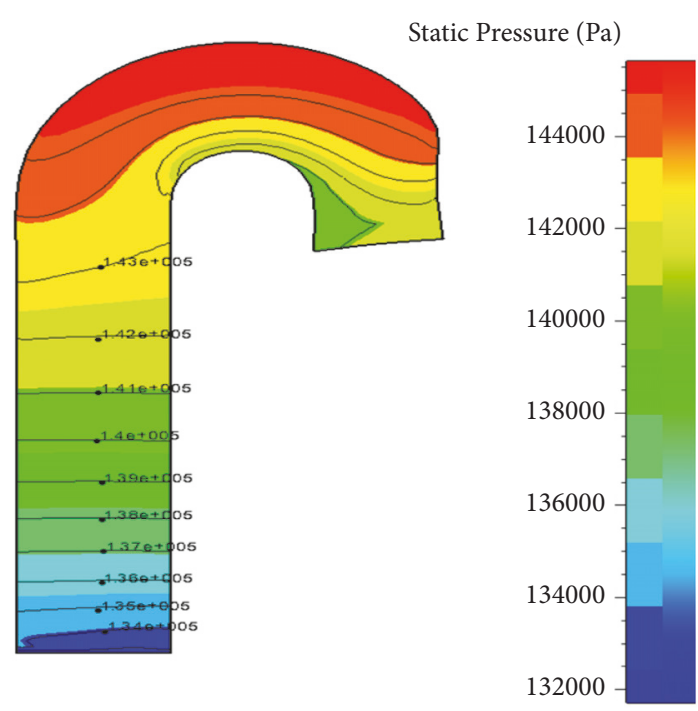

FIgURE 5: Static pressure distribution in the diffuser of the original compressor model.

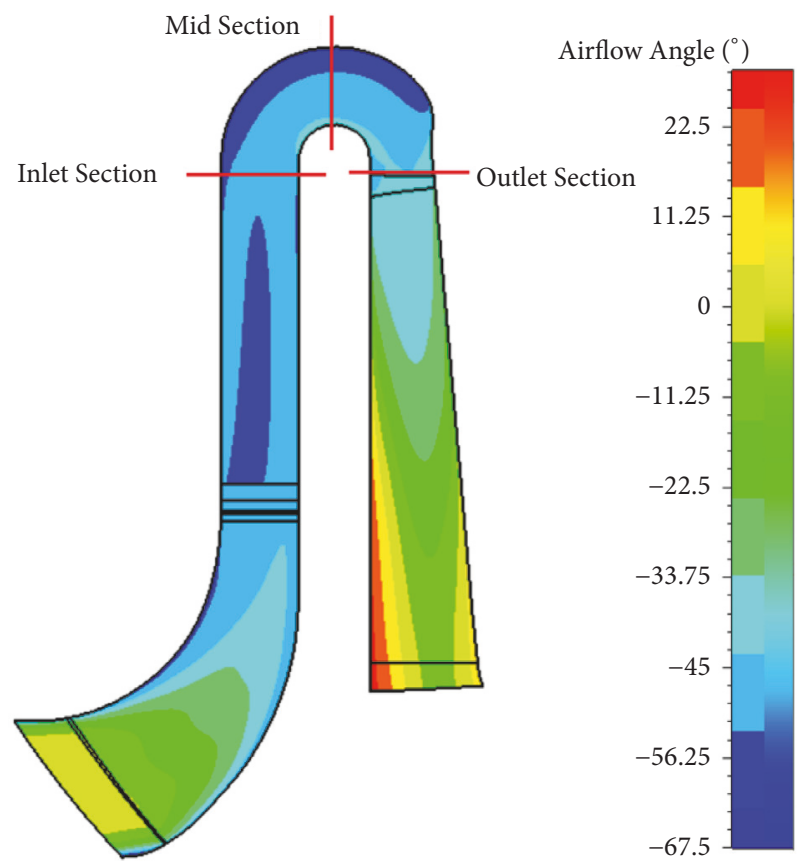

FIGURE 6: Distribution of the airflow angle in the meridional plane of the original compressor model.

(2) Inlet Design Angle of the Integrated Blade. Although in this paper optimization of the inlet design angle was performed using CFD techniques (see Section 4), some preliminary constraints affecting this parameter can be defined using results from previous literature. Specifically, we consider studies conducted by Johnston and Fleeter [13, 14], which state that the frequency of interaction between the blades should not coincide with the natural frequency of the impeller blade, to avoid resonance. Therefore, after determining the number of impeller blades, the number of integrated blades

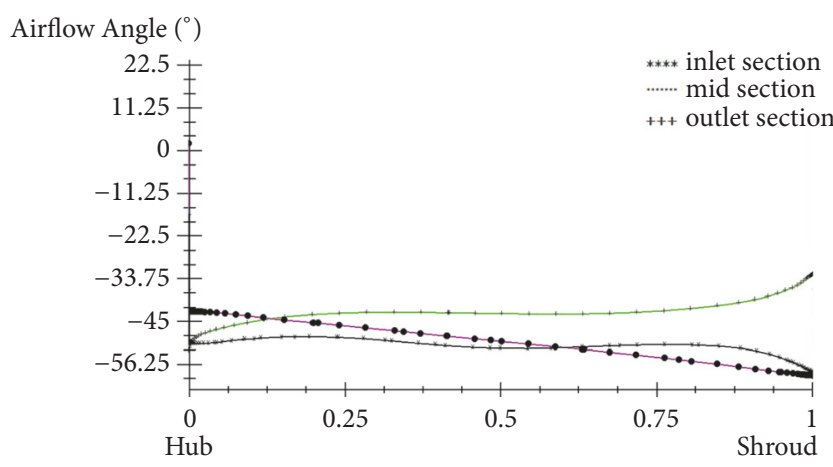

FIGURE 7: Variation of the airflow angle in each bend section of the original compressor model.

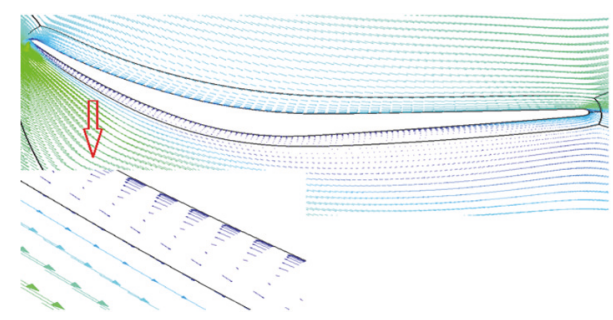

FIgURE 8: Velocity distribution in the return channel of the original compressor.

TABLE 3: Geometric parameters of the integrated blade.

\begin{tabular}{lc}
\hline Section & Value \\
\hline Blade number, $\mathrm{Z}$ & 10 \\
Speed, $\mathrm{N}(\mathrm{rpm})$ & 0 \\
Inlet diameter, $\mathrm{D}_{\text {in }}(\mathrm{mm})$ & 750 \\
Inlet width, $\mathrm{b}_{\text {in }}(\mathrm{mm})$ & 31.2 \\
Inner arc radius of bend, $\mathrm{R}_{1}(\mathrm{~mm})$ & 18 \\
Outer arc radius of bend, $\mathrm{R}_{2}(\mathrm{~mm})$ & 49.12 \\
Average outlet diameter, $\mathrm{D}_{\text {out }}(\mathrm{mm})$ & 466 \\
Average outlet width, $\mathrm{b}_{\text {out }}(\mathrm{mm})$ & 56.53 \\
\hline
\end{tabular}

should be the next highest prime number, to minimize the common multiples of these two sets of blades.

The integrated blade is designed to be of equal thickness throughout its volume, with a round leading edge and a blunt tail edge. The geometric parameters of this component are summarized in Table 3 . The inlet diameter, $D_{\text {in }}$, and the inlet width, $b_{i n}$, of the blade correspond to the $D_{3}$ and $b_{3}$ positions in Figure 10, respectively, while the average outlet diameter, $\mathrm{D}_{\text {out }}$, and the average outlet width, $\mathrm{b}_{\text {out }}$, correspond to the $\mathrm{D}_{6}$ and $\mathrm{b}_{6}$ positions. A three-dimensional model of the integrated blade and the corresponding computational model for the modified compressor are shown in Figures 11 and 12, respectively.

2.4. Grid Division. To generate the computation domain for analysis, the geometric models were first discretized using the Autogrid5 module in NUMECA. Following this, the 


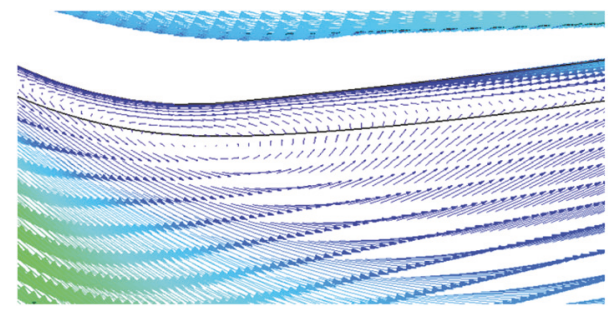

FIGURE 9: Vortex at the suction surface of the return channel in the original compressor.

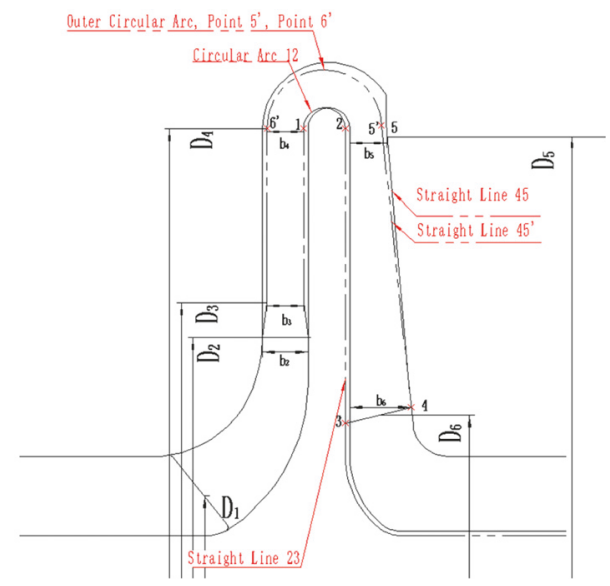

Figure 10: Meridional profile of the integrated blade. The solid line is the meridional line of the original model, and the dashed line is the meridional line of the new integrated blade.

program constructed a structured mesh in the $\mathrm{O} 4 \mathrm{H}$ grid topology. Prior to more detailed analysis, we first verified that the number of mesh elements were independent from the results of CFD analysis, to eliminate discretization errors. Figure 13 shows the relationship between the number of mesh elements and the airflow efficiency and pressure ratio calculated by the computational model. We note that when there were more than two million mesh elements, these parameters did not change significantly. Hence, the mesh can be considered to be independent beyond this point. To create a balance between accurate and speedy computation, the total number of mesh elements for a single channel was selected to be greater than three million. As we adopted a three-layer multigrid for the computational model used in this paper, the orthogonality, aspect ratio, and extension ratio of the mesh and mesh elements were defined to enable calculation in these conditions. Hence, the minimum orthogonal angle was greater than $15^{\circ}$, and the maximum aspect ratio and extension ratio were set to be less than 2000 and 5, respectively.

Figure 14 depicts a meshed channel, with 3,856,565 elements, in total. In this grid, the minimum orthogonal angle is $20.691^{\circ}$, the maximum aspect ratio is 1330.8 , and the maximum extension ratio is 3.5875 . This grid can subsequently be subdivided into an impeller section, consisting of 1,654,950 elements, with a minimum orthogonal angle of $28.583^{\circ}$, a maximum aspect ratio of 554.79 , and a maximum extension

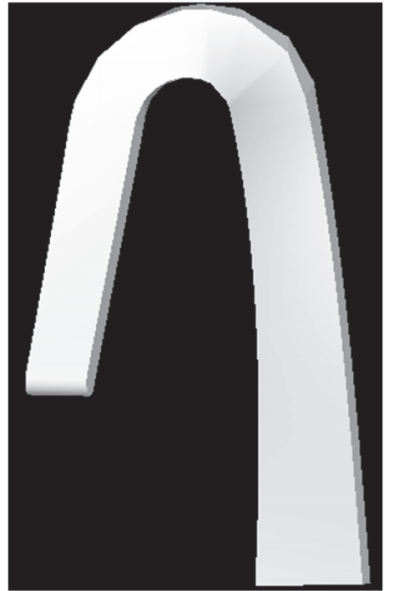

FIGURE 11: 3D model of the integrated blade.

ratio of 3.5875, and integrated blade section, consisting of $2,201,615$ elements, a minimum orthogonal angle of $20.691^{\circ}$, a maximum aspect ratio of 1330.8 , and a maximum extension ratio of 3.2457 .

In this model, we used a transpose on freezing method for connection between the rotor and stator. The wall thickness of the first layer is $0.01 \mathrm{~mm}$. The distribution of $\mathrm{y}^{+}$values on the solid wall is shown in Figure 15. This image demonstrates that the requirement that $\mathrm{y}^{+}$values are in the range of $1-10$, for the turbulence model, is satisfied.

\section{Calculation Method}

3.1. Numerical Methods. In this paper, we solved the model level flow field using NUMECA's FineTurbo module. The continuity, momentum (three dimensional Reynolds averaged Navier-Stokes equation), and energy equations are solved in calculation. These equations can be expressed in a general form of as defined in

$$
\frac{\partial(\rho \Phi)}{\partial t}+\nabla \cdot(\rho V \Phi)=\nabla \cdot\left(\Gamma_{\Phi} \nabla \Phi\right)+S_{\Phi}
$$

where $\rho$ is a density, $\mathrm{V}$ is a volume, $t$ is time, $\Phi$ is a general variable that can be substituted for a specific parameter, $\Gamma_{\Phi}$ is a generalized diffusion coefficient, and $S_{\Phi}$ is a generalized source term. Transient, convection, diffusion, and source terms can be identified from left to right in Equation (3). The computer updates this equation iteratively, to obtain the flow field for specific source term, diffusion term, and boundary condition combinations. Since in this paper, we consider a compressor in normal operation, (3) is solved in a steady-state condition.

In this paper, we adopt the grid topology structure developed in [15] for the solid model of a NACA model 404III centrifugal compressor. Using the FineTurbo program, we compared the results of computation using a zero equation (Baldwin-Lomax), one equation (Spalart-Allmaras) and a two equation (SST) turbulence model, to the experimental data provided in the literature, as shown in Figure 16. From 

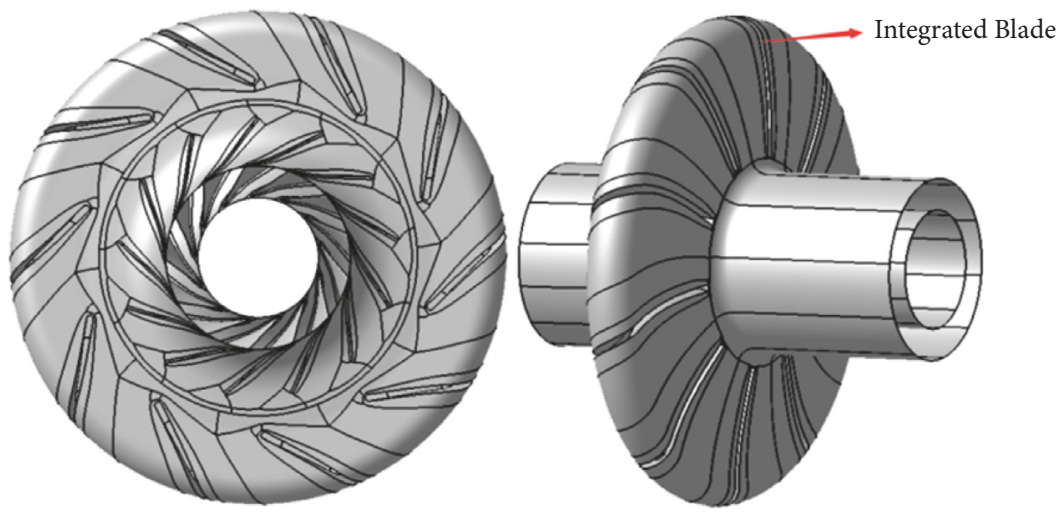

FIGURE 12: 3D model of the modified compressor.

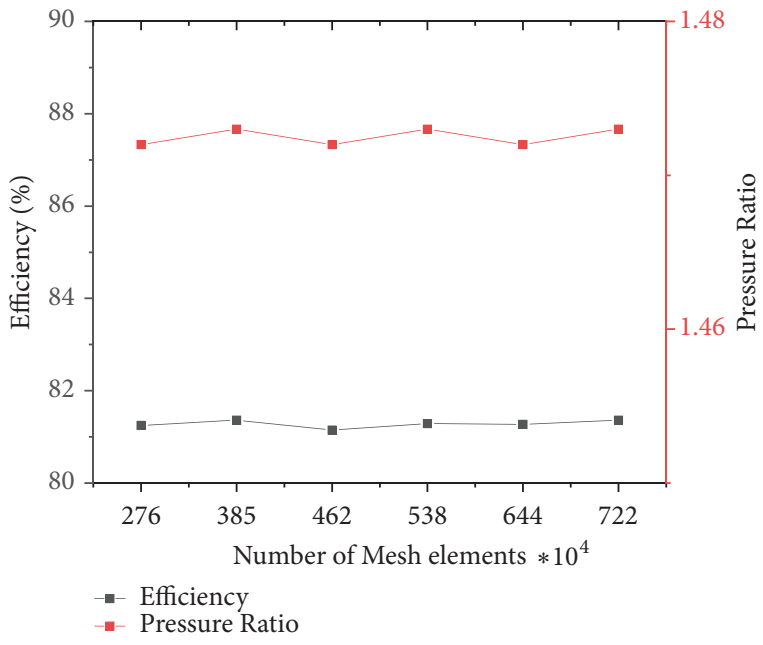

FIGURE 13: Relationship between the number of mesh elements, and predicted compressor level efficiency and pressure ratio when $\mathrm{D}_{\text {in }}=750 \mathrm{~mm}$.

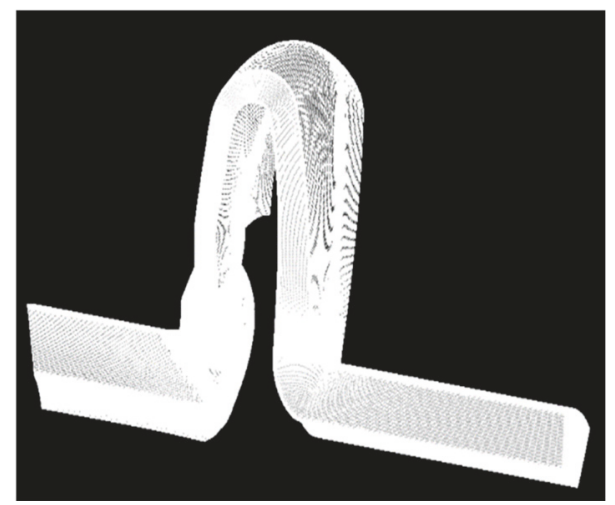

FIGURE 14: Computational grid for a single channel model.

these results, it can be seen that the pressure ratio curves calculated using the Spalart-Allmaras model are closest to values obtained from real tests. Similarly, although in the middle flow position the efficiency curve obtained from

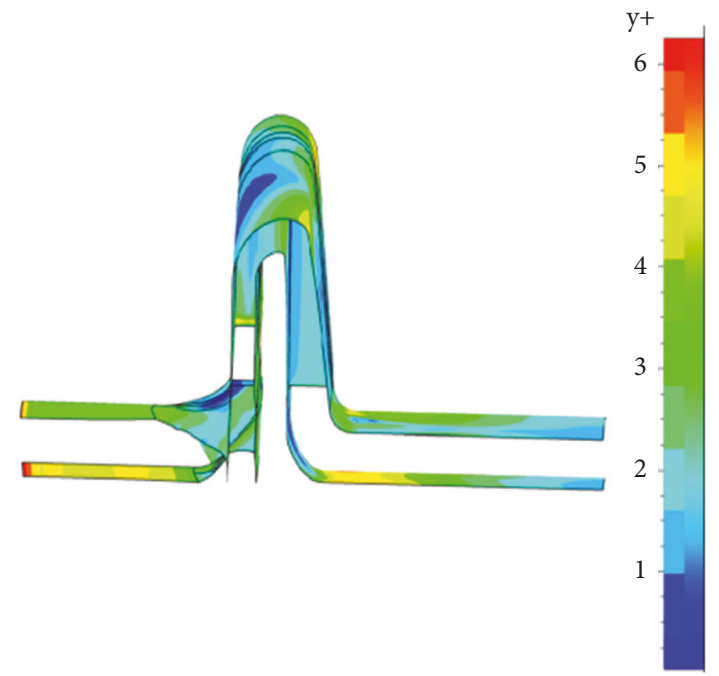

FIGURE 15: Distribution of $y+$ values on the solid walls of the model.

actual tests differs from the curves obtained from simulation, the results calculated using the Spalart-Allmaras model give the closest match to the results of practical experimentation. Hence, numerical simulation in this paper was completed using this turbulence model.

3.2. Boundary Conditions and Convergence Criteria. In the simulations discussed in this paper, the flowing fluid is modeled as air, a viscous, compressible gas. The interface of the rotator and the stator is depicted as a circumferential conserved connection surface. The CFL number for these simulations is 3 . The inlet boundary condition is in the direction of incoming flow, the total temperature and total pressure, and the outlet boundary condition are dictated by the mass flow rate, and outer boundaries of the computation domain are modeled as adiabatic walls with a constant rotational speed, in a no slip condition. All rotating parts have a specified rotational speed, while the rotational speed of the static component is set to zero. The simulated control parameters are reported following 6000 steps of iteration on 


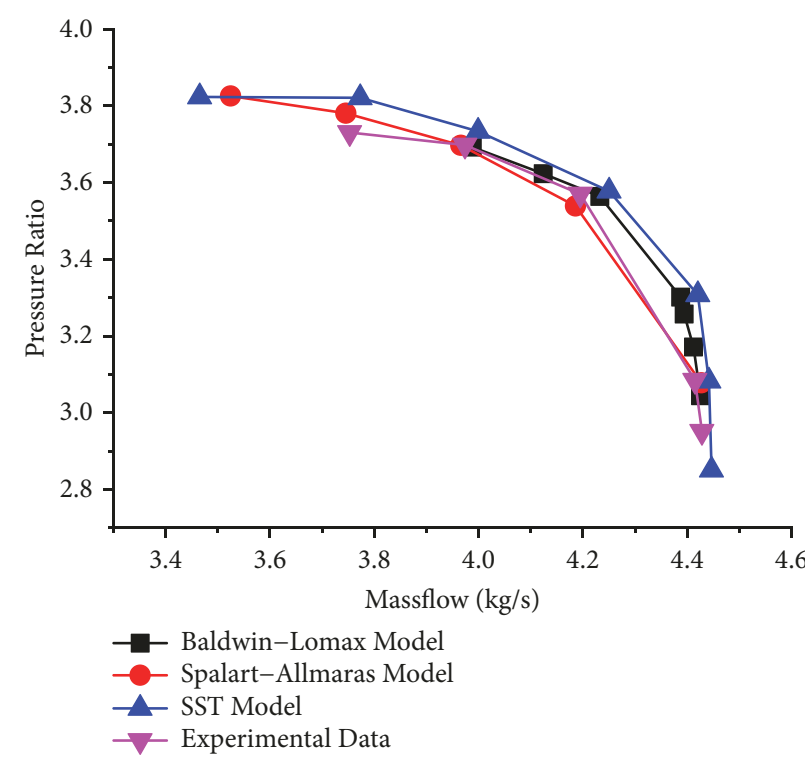

(a)

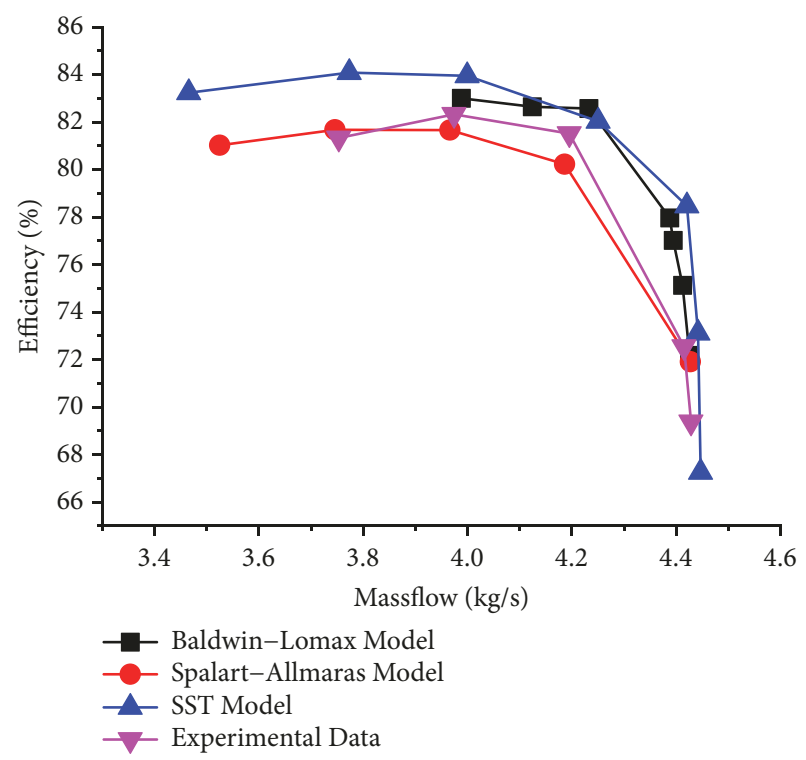

(b)

FIGURE 16: Comparison of the (a) pressure ratio and (b) compressor efficiency performance curves predicted using different turbulence models, with experimental data.

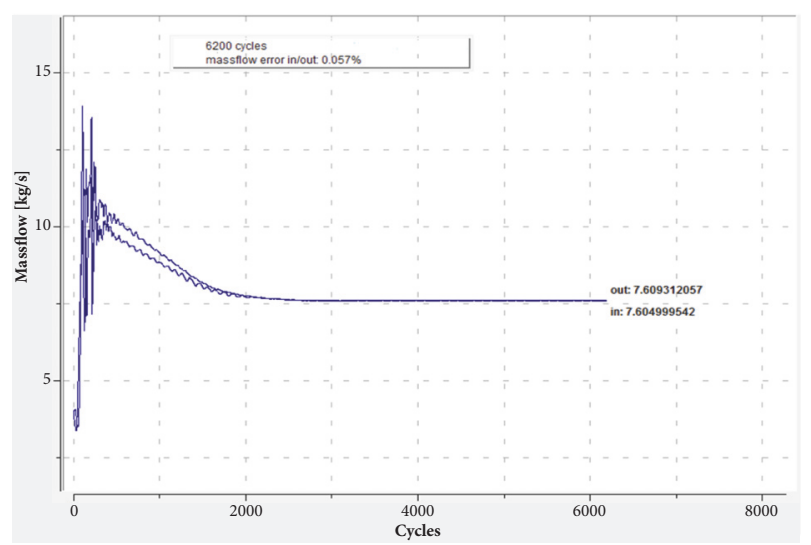

FIGURE 17: Convergence history for a compressor model with $\mathrm{D}_{\text {in }}=750 \mathrm{~mm}$.

the finest grid, or when convergence residuals are of the order of $10^{-6}$.

Convergence criteria are determined as follows: global residuals and residuals in each block has decreased by more than three orders of magnitude; relative error of import and export mass flow is less than $0.5 \%$ and the flow rate is no longer changed; the values of the control parameters at each point of the flow field remain unchanged; or the overall performance (determined using a combination of parameters including the efficiency, torque, thrust) does not vary with the number of iterations [16]. Figure 17 shows a convergence curve for an integrated blade $\left(D_{\text {in }}=750 \mathrm{~mm}\right)$ model at the design point, illustrating when calculation has been completed.

\section{Results and Discussion}

4.1. Influence of Inlet Design Angle on Integrated Blade. The inlet design angle of the integrated blade has a large effect on airflow efficiency, as a suitable inlet design angle can minimize angle of incidence of airflow, and reduce flow loss, improving the efficiency of the whole compressor stage. Hence, in this paper, we focus on controlling this angle in designing the integrated blade. By establishing geometric models of different inlet design angles and analyzing the flow field, a solution that optimizes the efficiency of the compressor stage can be obtained. The inlet design angle of the integrated blade was determined according to angle of the gas flowing out of the impeller. In order to make the gas flow through the blade into the lower stage of the impeller uniformly, the outlet angle was set to $0^{\circ}$. The inlet design angle was defined as the angle between the tangent of the leading edge of the middle arc of the blade and the horizontal axis.

Figure 18 shows the stage efficiency and pressure ratio curves of the compressor model with different inlet design angles. From this diagram, it can be observed that as the inlet design angle increases, both the efficiency and the pressure ratio of the model first increase to a maximum at $\sim 62^{\circ}$ (78.28\% and 1.447, respectively), and decrease subsequently. The change in efficiency when the inlet design angle is between $62^{\circ}$ and $66^{\circ}$, is more gradual, giving room for error in selecting this parameter, without affecting the performance of the compressor.

To demonstrate the effect of inlet design angle on the performance of the integrated blade in further detail, we have illustrated the flow characteristics of gas inside the compressor at design angles of $73^{\circ}, 62^{\circ}$, and $50^{\circ}$. Figure 19 shows the total pressure distribution of the meridional section at these inlet angles. Our analysis shows that, at a design angle of 


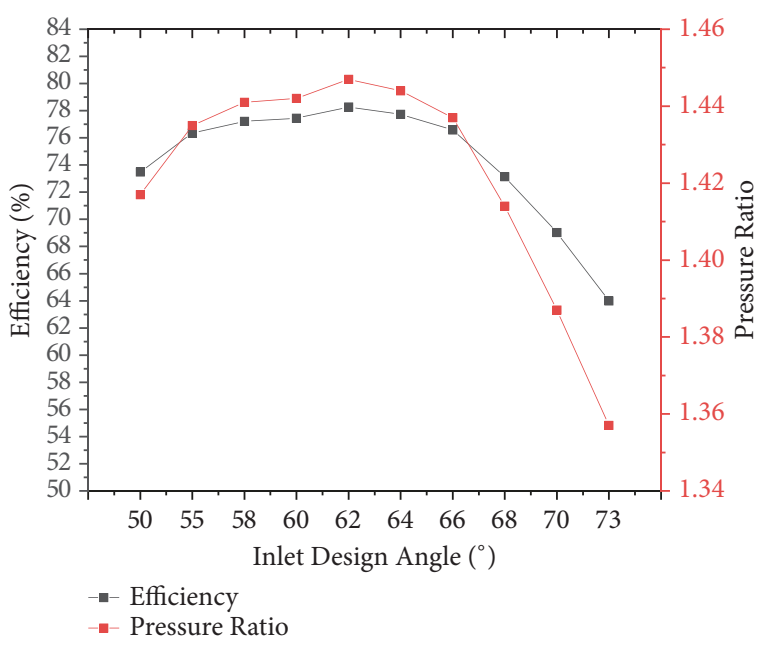

FIGURE 18: Compressor efficiency and pressure ratio of the modified model as a function of the integrated blade's inlet design angle.

$73^{\circ}$, there is a pressure loss close to the inlet of the blade passage, and there is a noticeable low pressure section in the mainstream region. After the bend, a pressure gradient is formed from the shroud of the wheel to the hub, resulting in a large pressure drop. The isobaric lines are densely packed until the return channel, and the pressure distribution remains uneven. When the inlet design angle is $62^{\circ}$, the pressure distribution at the inlet is uniform, and pressure loss in the diffuser passage disappears. Although pressure loss through the bend is inevitable even in this condition due to the effects of centrifugal force, in general, the pressure distribution remains uniform, and the loss is reduced in comparison to that observed with other design angles. The pressure distribution observed with an inlet design angle of $50^{\circ}$ is similar to that depicted in Figure 19(a) (where the inlet design angle is $73^{\circ}$ ), and the low pressure region in the diffuser channel reappears.

Figure 20 shows the distribution of velocity vectors for the different inlet design angles, with the diagrams enlarged from their original scale by an additional $50 \%$. As suggested by Figure 18, energy loss-inducing return and vortex phenomena are not observed with a design angle of $62^{\circ}$ (Figure 20(d)). In contrast, with a design angle of $73^{\circ}$, the gas separation phenomenon at the inlet side of the blade is increased, due to inconsistencies between the airflow and the blade direction. Air return and vortices are consequently generated, resulting in a large amount of energy loss (Figure 20(a)). This loss of energy stalls airflow at the suction side of the surface, downstream of the blade, also resulting in the occurrence of return and vortex phenomena (Figure 20(b)), which further compounds the reduction in compressor efficiency. These phenomena disappear with an inlet design angle of $50^{\circ}$ (Figure 20(c)), there is a good flow of air on the pressure side of the integrated blade, and gas return and vortex phenomenon disappear, corresponding to the comparatively improved efficiency (with respect to the performance with a $73^{\circ}$ design angle) of the compressor in this condition, previously suggested in Figure 18.
4.2. Effect of Radial Spacing between Rotor and Stator on Stage Performance. Optimizing the interaction between moving and static components plays an important role in improving the performance of a centrifugal compressor. In our model, this interaction occurs in the region between the tail of the impeller and the leading edge of the integrated blade. In this paper, only the distance between moving and static components is modified to affect this interaction, as the inlet design angle and the diameter of the tail edge of the integrated blade are fixed. We conducted simulations with radial spacings (R) of $15 \mathrm{~mm}, 30 \mathrm{~mm}, 45 \mathrm{~mm}, 60 \mathrm{~mm}, 75$ $\mathrm{mm}, 90 \mathrm{~mm}$, and $105 \mathrm{~mm}$. Since all the other geometric parameters of the compressor were fixed, the length of the integrated blade decreases as $\mathrm{R}$ is increased, as illustrated in Figure 21.

Figure 22 shows the variation of compressor efficiency and pressure ratio with radial spacing. These parameters increase to maximums of $81.36 \%$ and 1.473 , respectively, as $\mathrm{R}$ is increased to $75 \mathrm{~mm}$. Further increasing the radial spacing beyond this point decreases the efficiency of the compressor.

To demonstrate the effect of radial distance on the flow field of the impeller blade and the integrated blade, we have included diagrams illustrating the distribution of total pressure, static pressure, air flow angle, and velocity, with $\mathrm{R}=15 \mathrm{~mm}, 45 \mathrm{~mm}$, and $60 \mathrm{~mm}$.

Figures 23 and 24 show the total pressure and static pressure distribution at the meridional surface of the integrated blade, respectively. We note decreases in the total pressure in the diffuser channel and the bend as the radial spacing is decreased, a phenomenon which is most notable when $\mathrm{R}=15 \mathrm{~mm}$. In addition, the reduction in total pressure loss is larger at the hub. This is because, as previously mentioned, the length of the integrated blade increases as the radial spacing decreases, which increases the magnitude of friction loss on the airflow in the passage. No significant differences in the reduction in total pressure loss were observed with radial spacings of 45 and $60 \mathrm{~mm}$. The static pressure of the compressor stage also increased with these radial spacings (see Figure 24), further highlighting the effect of this distance on airflow.

Figures 25 and 26 show the distribution of airflow angles at the outlet of the impeller blade and the inlet of the integrated blade, respectively. From our simulations, when $\mathrm{R}=15 \mathrm{~mm}$, the flow angle distribution is uneven in both regions considered. This demonstrates that when the distance between moving and static components is too small, the flow field downstream of the impeller blade has an effect upstream of this component; the interaction between moving and static components makes airflow at the outlet of impeller blade uneven. At the same time, the airflow angle at the inlet of the integrated blade is also uneven due to the influence of impeller wake. Since increasing the radial spacing decreases impeller wake, the uniformity of the airflow angle distribution is marginally improved.

Figure 27 shows the distribution of velocity vectors for the different radial spacings, with the diagrams enlarged by an additional $50 \%$. When $\mathrm{R}=15 \mathrm{~mm}$, we note a gas separation phenomenon on the surface of the blade at the inlet side, 


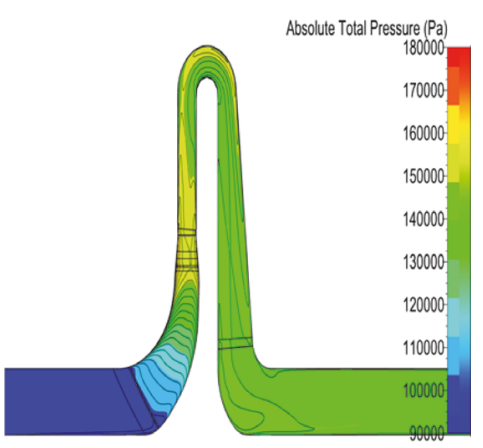

(a)

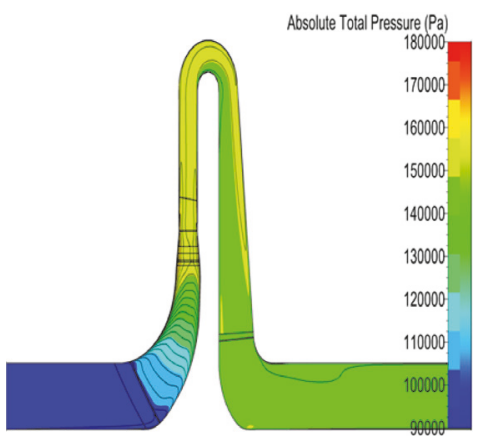

(b)

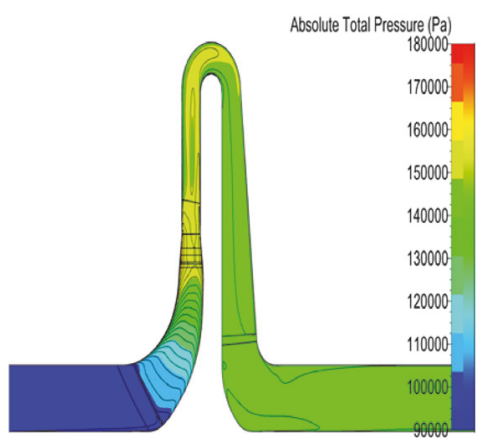

(c)

FIGURE 19: Total pressure distribution at the meridional surface of the modified compressor model with integrated blade inlet design angles at (a) $73^{\circ}$, (b) $62^{\circ}$, and (c) $50^{\circ}$.

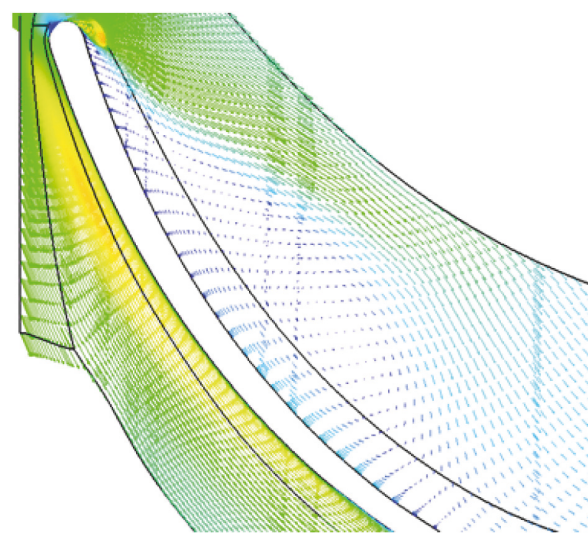

(a)

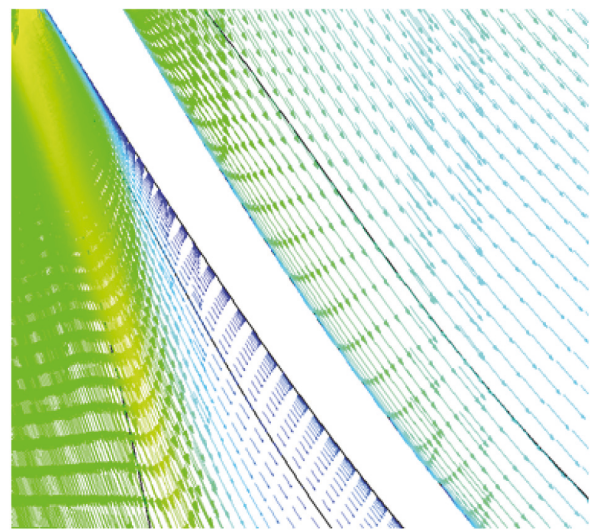

(c)

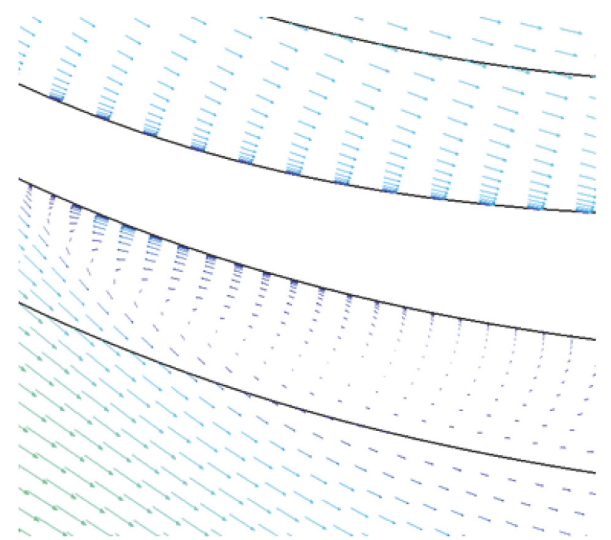

(b)

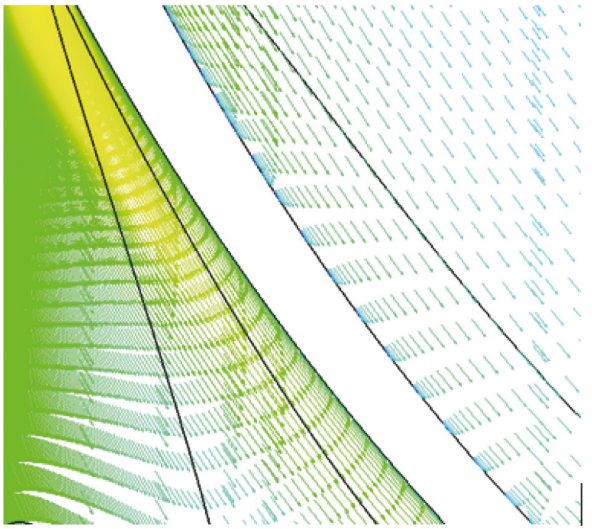

(d)

Figure 20: Velocity vectors (enlarged by an additional 50\%) for selected integrated blade inlet design angles and surfaces. (a) Integrated blade's inlet pressure surface at $73^{\circ}$. (b) Integrated blade's suction surface at $73^{\circ}$. Integrated blade's inlet pressure surface at (c) $50^{\circ}$ and (d) $62^{\circ}$.

which results in the generation of vortex and reflux motion. This separation is caused by the uneven flow of air from the impeller, an explanation obtained through analysis of the airflow angle distribution. Increasing the radial spacing weakens the effect of impeller blade wake, and the air flow becomes uniform. Consequently, the gas separation and vortex phenomena disappear.
4.3. Analysis and Evaluation of Integrated Blade Internal Flow. To illustrate the improvement in compressor efficiency achieved with our modifications, we have included diagrams of the total and static pressure distributions in the original compressor model, and a model with $\mathrm{D}_{\text {in }}=750 \mathrm{~mm}(\mathrm{R}=75$ $\mathrm{mm}$ ) (See Figures 28 and 29). We have also included diagrams of the airflow angle distribution in each section of the bend, 


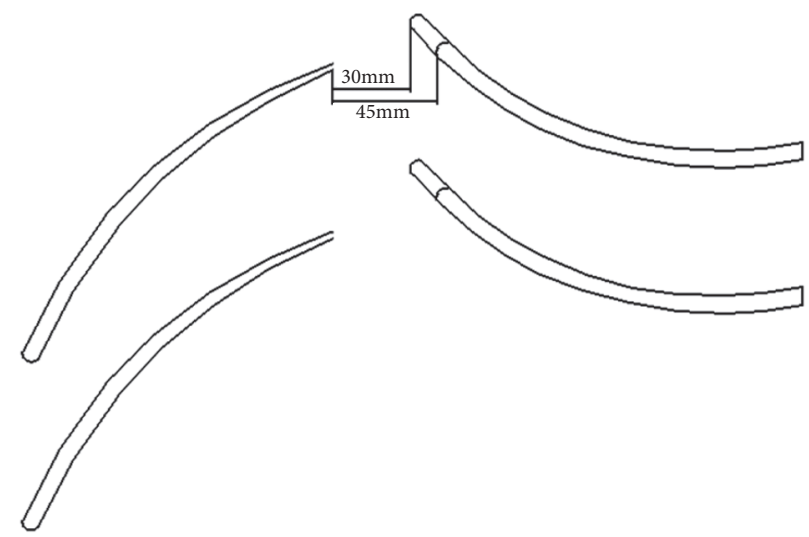

FIGURE 21: Effect of modifying the distance between moving and static components on blade structure.

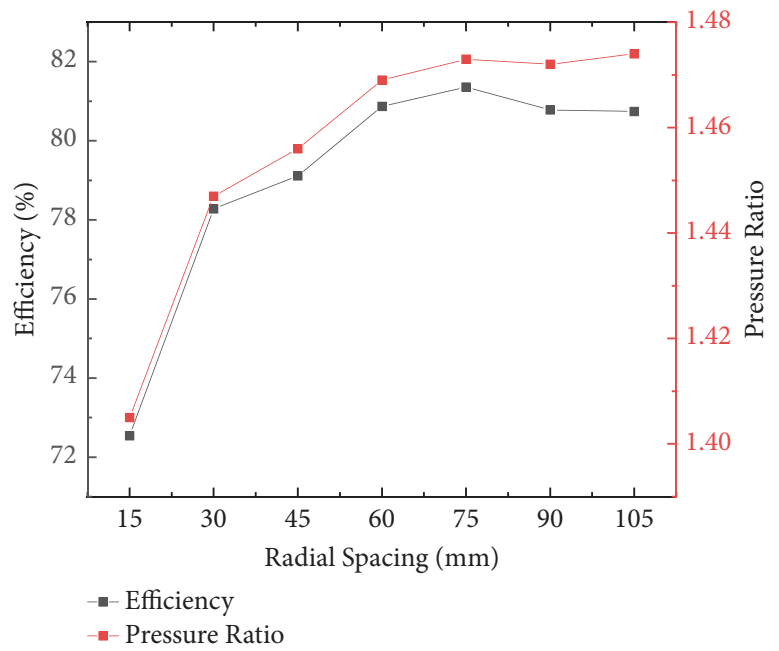

FiguRE 22: Variation of compressor level efficiency and pressure ratio with the radial spacing between moving and static components.

and the distribution of velocity vectors in the passage, with these diagrams enlarged by an additional $50 \%$ (see Figures 30 and 31).

Figures 28 and 29 highlight that, with the improved model, the total pressure loss in the boundary layer of the diffuser channel, especially in the mainstream of the return channel, has been decreased. We also note that the static pressure of the improved model has been enhanced, based on the more uniform isoline distribution.

Figure 30 shows the distribution of airflow angles in each section where gas flows in the bend. In the original model, the flow direction changes as the gas turns in the bend. Hence, although the airflow angle at the inlet section is uniform, this is not the case at the outlet section and gas flow is separated. Installing an integrated blade in the bend of the compressor guides airflow effectively, such that the airflow angle in the outlet section of the bend is more uniform in the improved model, reducing the occurrence of the separation phenomenon.

Figure 31 shows the velocity distribution on the integrated blade, and the flow field of the original model. In the latter, the uneven airflow distribution impinging on the return blade results in separation loss at the inlet suction surface of the return blade; a clear vortex region is formed downstream of the blade as air moves. No airflow stall phenomenon is observed at the inlet, and downstream of the integrated blade, with the improved compressor. In addition, there are no reflux and separation phenomena, indicating that this integrated blade design has a good guiding effect on airflow.

Table 4 summarizes the total pressure loss coefficient for each section, before and after including the modifications. From this, it can be seen that including an integrated blade reduced the total pressure loss coefficient of the return channel (which originally had the largest decrease in total pressure) by $64.34 \%$. Similarly, this coefficient was decreased by $30.34 \%$ and $16.67 \%$, respectively, for the diffuser channel (moderate total pressure loss) and the bend section (minimum total pressure loss). The static pressure recovery coefficient of the diffuser section is increased by $28.39 \%$. With these modifications, the efficiency of the compressor stage is $81.36 \%$, and the pressure ratio is 1.473 , which constitutes a $4.39 \%$ increase to the efficiency of the original model $(76.97 \%)$, and a $2.86 \%$ increase to its pressure ratio (1.432). 


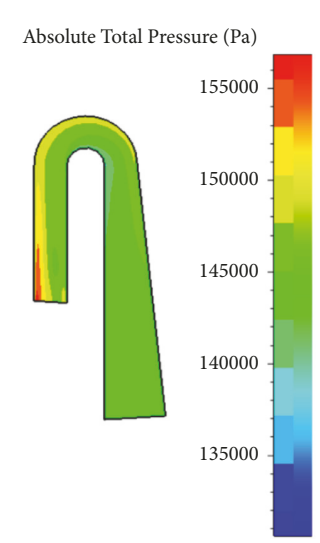

(a)

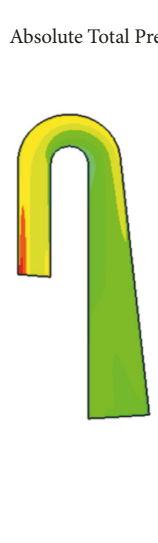

(b)

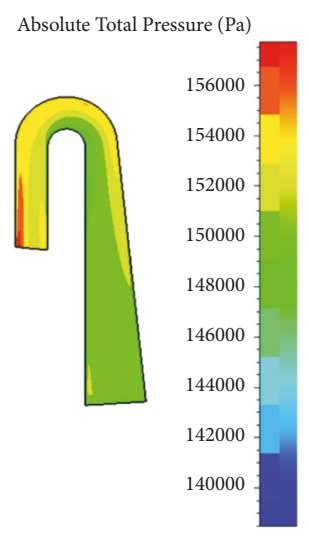

(c)

FiguRE 23: Total pressure distribution at the meridional plane of the integrated blade. (a) $\mathrm{R}=15 \mathrm{~mm}$, (b) $\mathrm{R}=45 \mathrm{~mm}$, and (c) $\mathrm{R}=60 \mathrm{~mm}$.

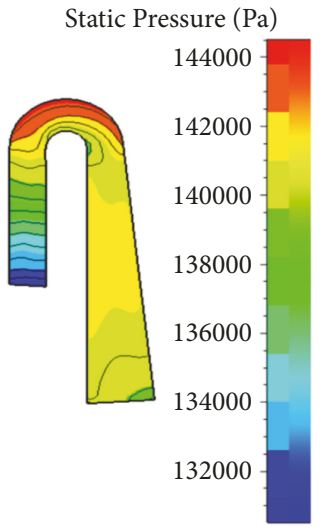

(a)

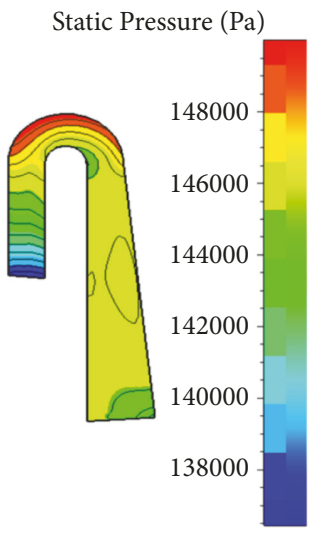

(b)

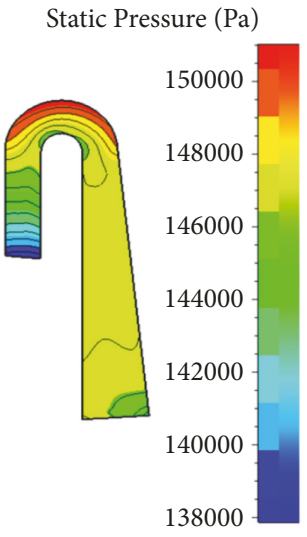

(c)

FIgURE 24: Static pressure distribution at the meridional plane of the integrated blade. (a) $\mathrm{R}=15 \mathrm{~mm}$, (b) $\mathrm{R}=45 \mathrm{~mm}$, and (c) R=60 mm.

\section{Conclusions}

In this paper, we have conducted numerical modeling of the intermediate stage of a multistage centrifugal compressor used in practical engineering, using CFD techniques. The flow field in this stage was simulated, and flow loss mechanisms were analyzed by considering total pressure loss in each of the diffuser, the bend, and the return channels in detail. This analysis suggested that including an integrated blade connected to these three channels would be advantageous in improving airflow in the compressor. A number of conclusions were obtained in designing and optimizing this integrated blade. We noted that the inlet design angle of the integrated blade affects the flow of gas and the overall efficiency of the compressor. The optimal design angle is at $62^{\circ}$, as the compressor efficiency and pressure ratio are maximized at this point. Although modifications to this optimal angle (increases and decreases) decrease both the pressure ratio and the efficiency, there are only slight decreases to the latter parameter when the design angle is between $62^{\circ}$ and $66^{\circ}$, giving room for error with this constraint. In addition, with this model, the optimal radial spacing between rotor and stator components is $75 \mathrm{~mm}$, as the pressure ratio and efficiency are maximized at this point. Reducing this radial distance increases the length of the integrated blade, and increases friction loss. At the same time, inhomogeneous airflow, caused by impeller blade wake, produces impact and separation phenomenon at the inlet of the integrated blade, also resulting in energy loss.

A comparison of the performance of the original compressor with that of a model including the above modifications, performed using numerical simulation, proves that an integrated blade is efficient in guiding the flow of gas in the compressor, improving the uniformity of the gas flow angle in the bend and reducing flow loss in the fixed elements. The combined result of these effects is to improve the efficiency of the modified model by up to $4.39 \%$ in the simulated operating condition, and the pressure ratio by $2.86 \%$, relative to the original compressor. This numerical analysis proves the feasibility of including integrated blades for fixed elements in a multistage centrifugal compressor. The concepts encountered in this paper can act as a reference for future research of interstage components of multistage centrifugal compressors. 


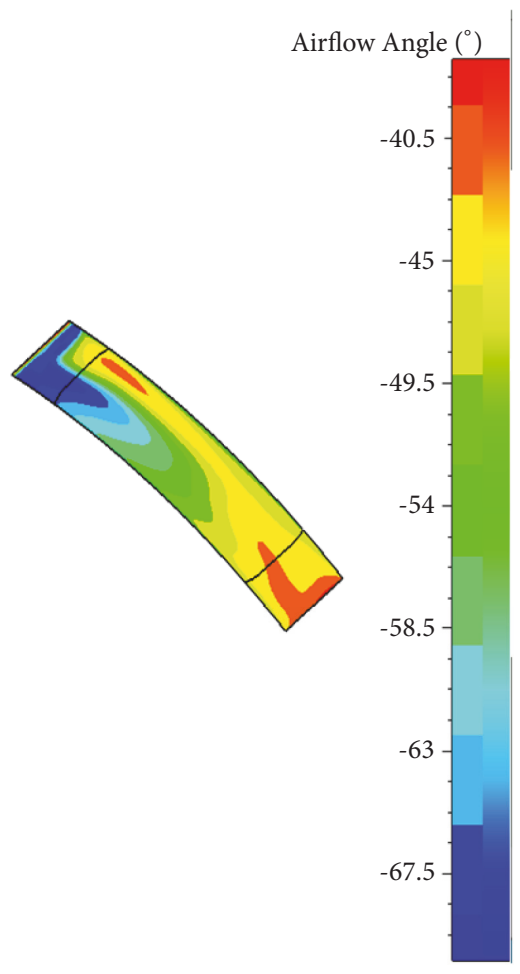

(a)

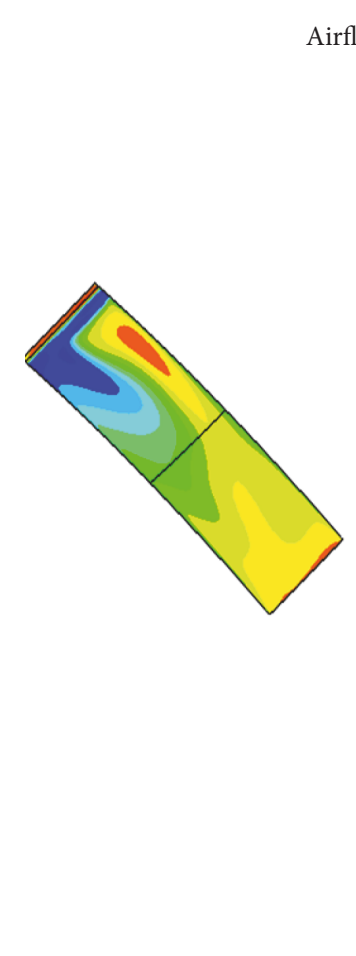

(b)

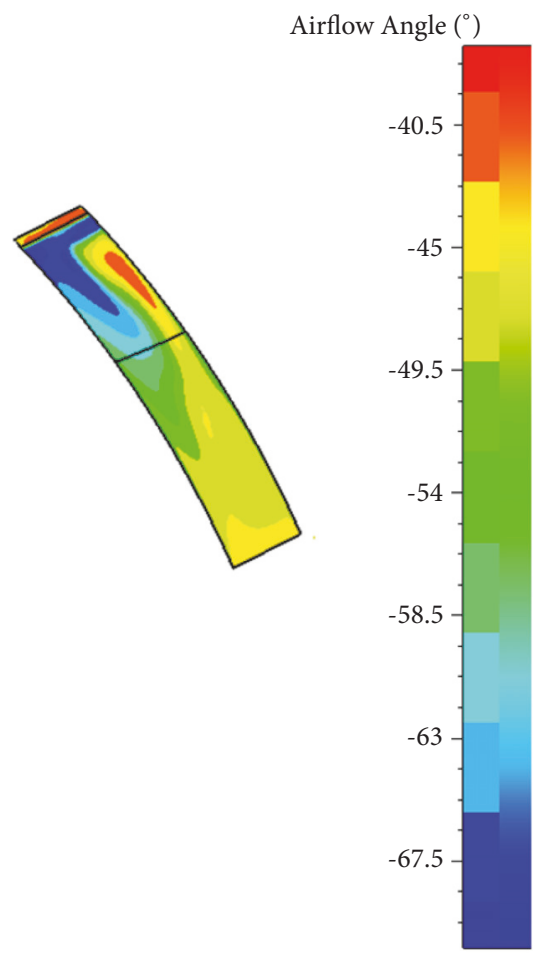

(c)

Figure 25: Airflow angle distribution at the outlet section of impeller blade. (a) $\mathrm{R}=15 \mathrm{~mm}$, (b) $\mathrm{R}=45 \mathrm{~mm}$, and (c) $\mathrm{R}=60 \mathrm{~mm}$.

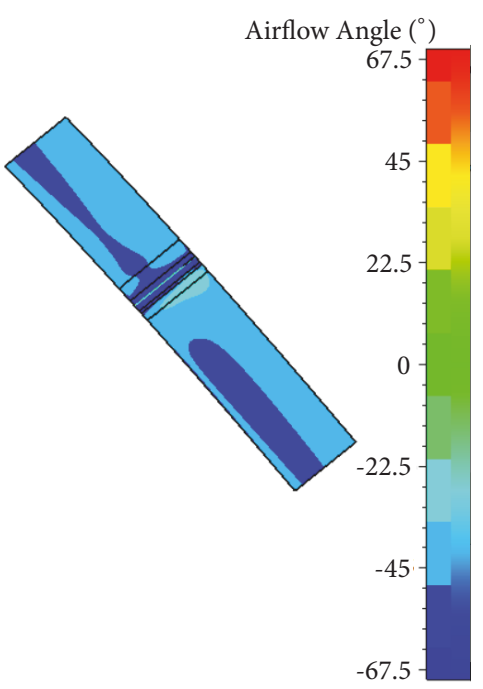

(a)

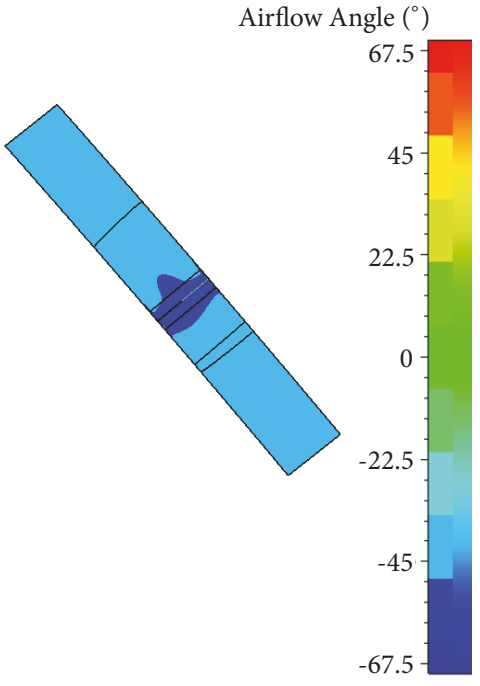

(b)

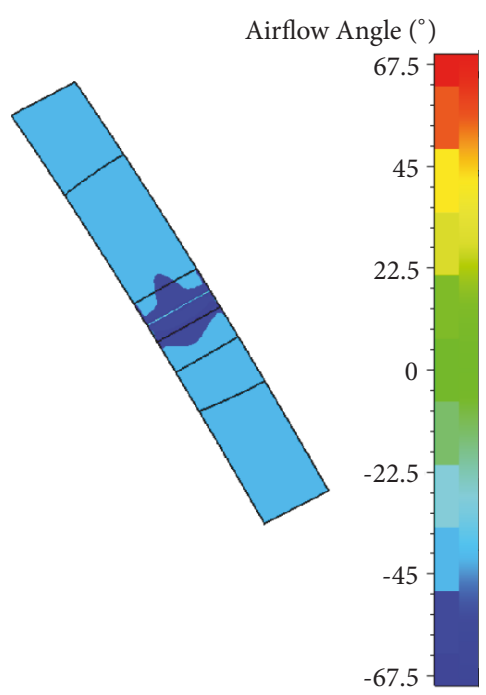

(c)

Figure 26: Airflow angle distribution at the inlet section of the integrated blade. (a) $\mathrm{R}=15 \mathrm{~mm}$, (b) $\mathrm{R}=45 \mathrm{~mm}$, and (c) $\mathrm{R}=60 \mathrm{~mm}$.

TABLE 4: Total pressure loss coefficient for each compressor section before and after modification.

\begin{tabular}{lccc}
\hline Section & Before modification & After modification & Percentage (\%) \\
\hline Total pressure loss coefficient of diffuser channel & 0.089 & 0.062 & 30.34 \\
Static pressure recovery coefficient of diffuser channel & 0.479 & 0.615 & 28.39 \\
Total pressure loss coefficient of bend channel & 0.072 & 0.060 & 16.67 \\
Total pressure loss coefficient of return channel & 0.129 & 0.046 & 64.34 \\
\hline
\end{tabular}




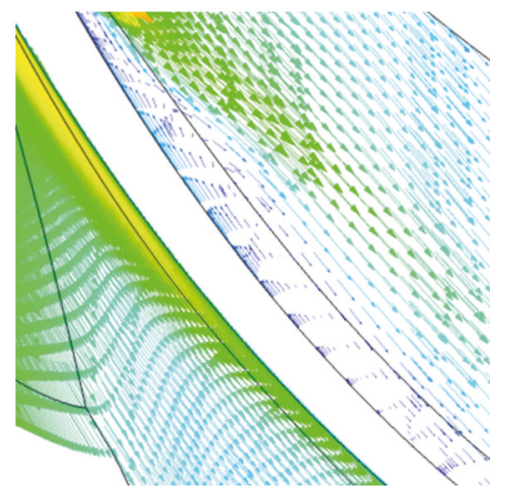

(a)

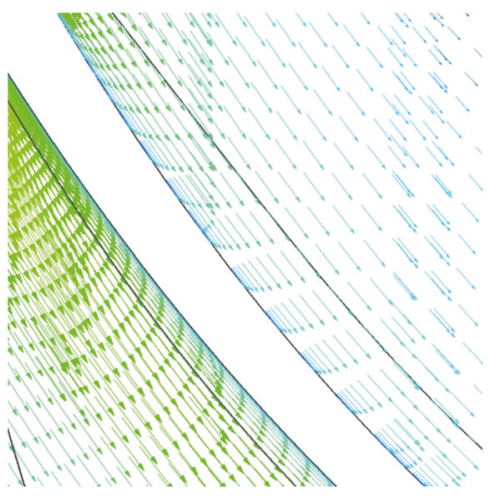

(b)

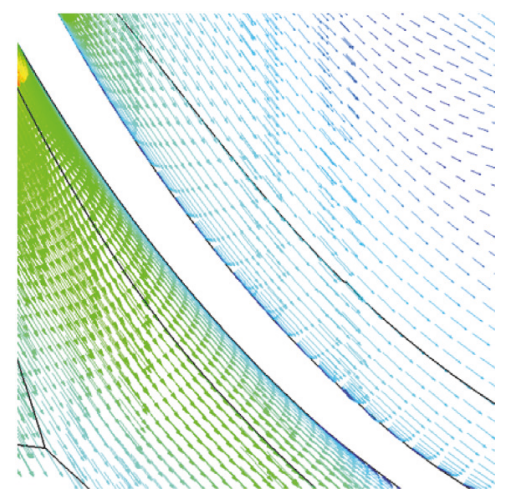

(c)

FIgURE 27: Enlarged (50\%) map of the velocity distribution at the integrated blade. (a) R=15 mm, (b) R=45 mm, and (c) R=60 mm.

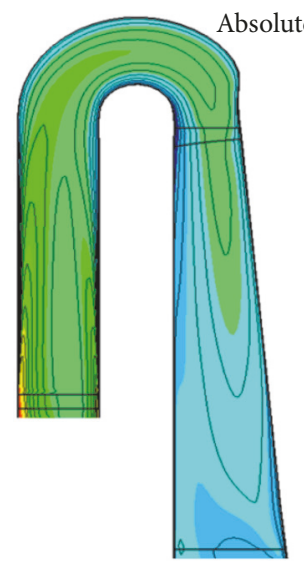

(a)
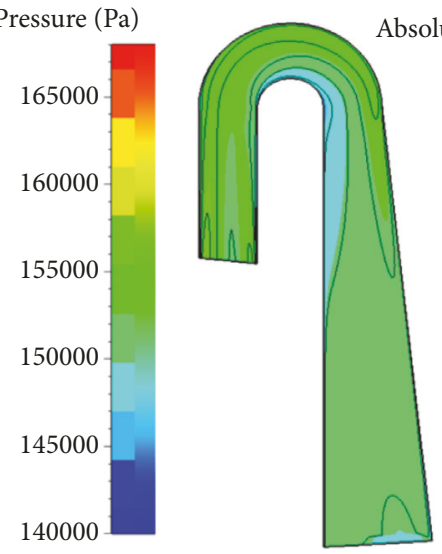

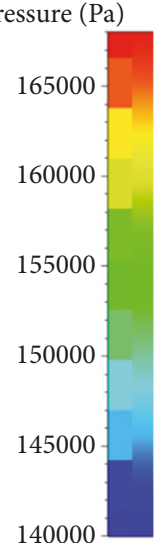

(b)

FIGURE 28: Total pressure distribution at the meridional plane of the (a) original model and (b) improved model.

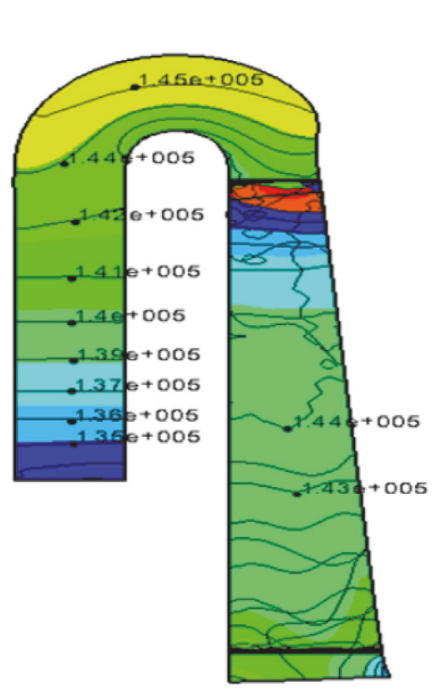

(a)
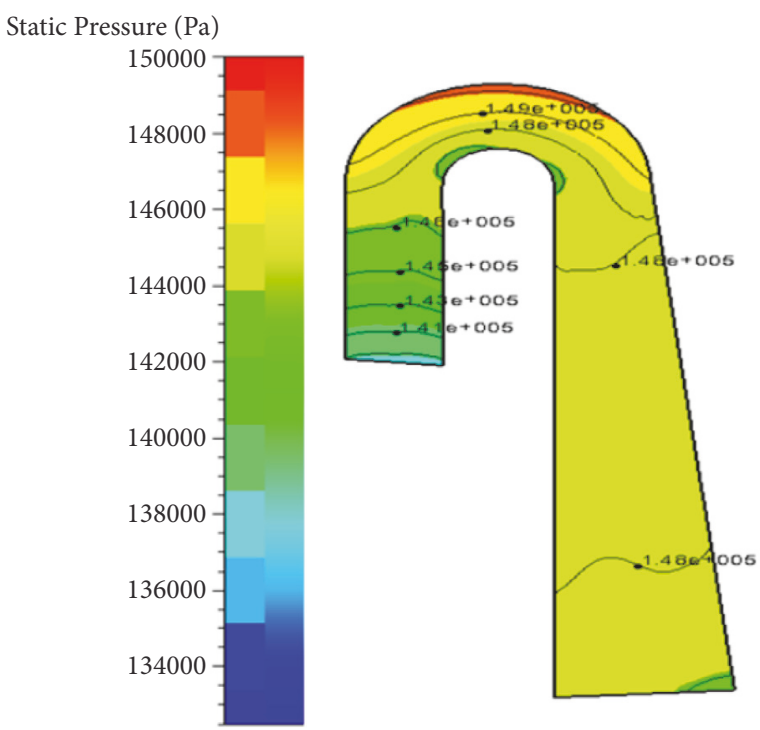

(b)
Static Pressure (Pa)

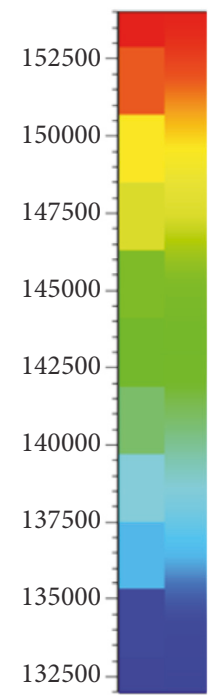

FIGURE 29: Static pressure distribution at the meridional surface of the (a) original mode, and (b) improved model. 


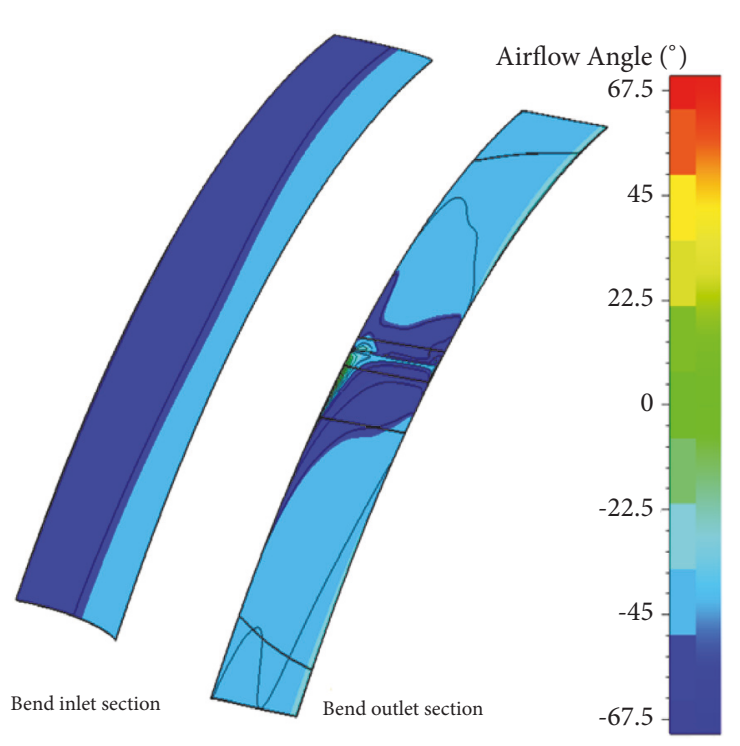

(a)

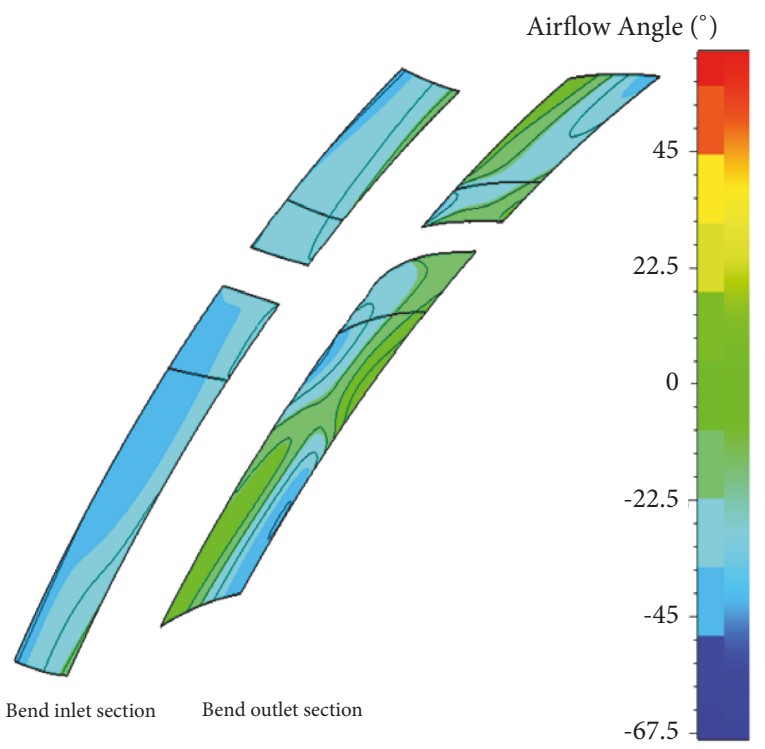

(b)

FIGURE 30: Distribution of airflow angles in each section of the bend in the (a) original model and (b) improved model.

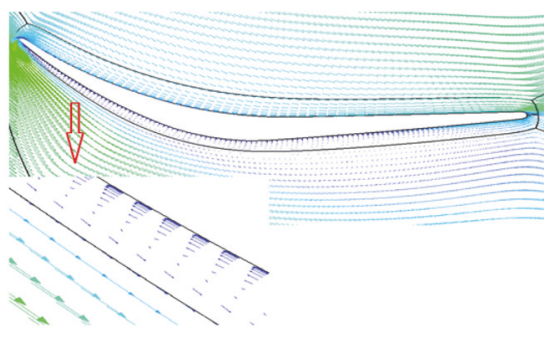

(a)

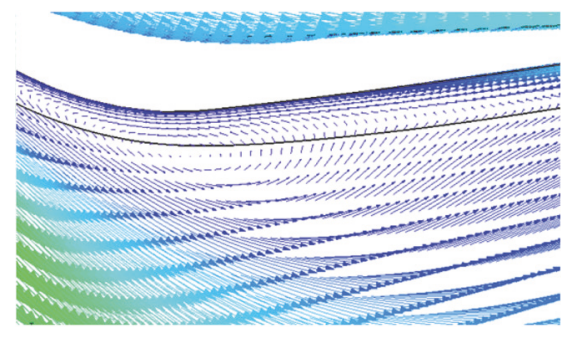

(b)

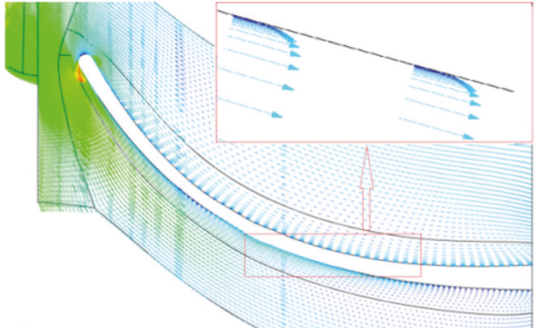

(c)

Figure 31: Velocity distribution of the internal flow field in the different compressors. (a) Return channel in the original model. (b) Vortex at the suction surface in the return channel of the original model. (c) Integrated blade in the improved model.

\section{Data Availability}

The data used to support the findings of this study are available from the corresponding author upon request.

\section{Conflicts of Interest}

The authors declare that there are no conflicts of interest regarding the publication of this paper.

\section{References}

[1] Y. Wang, D. Shi, D. Zhang, and Y. Xie, "Investigation on unsteady flow characteristics of a SCO2 centrifugal compressor," Applied Sciences (Switzerland), vol. 7, no. 4, 2017.

[2] V. G. Filipenco, S. Deniz, J. M. Johnston, E. M. Greitzer, and N. A. Cumpsty, "Effects of inlet flow field conditions on the performance of centrifugal compressor diffusers: Part 1discrete-passage diffuser," Journal of Turbomachinery, vol. 122, no. 1, pp. 1-10, 2000.
[3] D. Bonaiuti, A. Arnone, C. Hah, and H. Hayami, "Development of secondary flow field in a low solidity diffuser in a transonic centrifugal compressor stage," in Proceedings of the ASME Turbo Expo 2002: Power for Land, Sea, and Air, pp. 445-454, Amsterdam, The Netherlands, 2002.

[4] P.-F. Zhao, Y. Liu, and X.-F. Wang, "Analysis of vortices and performance of different diffusers in a large mass flow coefficient centrifugal compressor," Proceedings of the Institution of Mechanical Engineers, Part A: Journal of Power and Energy, vol. 231, no. 4, pp. 253-273, 2017.

[5] P. Zhao, Y. Liu, H. Li, X. Wang, and J. Yang, "The effect of impeller-diffuser interactions on diffuser performance in a centrifugal compressor," Engineering Applications of Computational Fluid Mechanics, vol. 10, no. 1, pp. 565-577, 2016.

[6] A. Hildebrandt and F. Schilling, "Numerical and experimental investigation of return channel vane aerodynamics with twodimensional and three-dimensional vanes," Journal of Turbomachinery, vol. 139, no. 1, 2017.

[7] M. Hosseini, Z. Sun, X. He, and X. Zheng, "Effects of radial gap ratio between impeller and vaned diffuser on performance of centrifugal compressors," Applied Sciences, vol. 7, no. 7, p. 728, 2017. 
[8] H. Tamaki, "Experimental study on the effect of diffuser vane setting angle on centrifugal compressor performance," Journal of Turbomachinery, vol. 139, no. 6, 2017.

[9] M. Ubaldi, P. Zunino, G. Barigozzi, and A. Cattanei, "An experimental investigation of stator induced unsteadiness on centrifugal impeller outflow," Journal of Turbomachinery, vol. 118, no. 1, pp. 41-51, 1996.

[10] Y. K. P. Shum, C. S. Tan, and N. A. Cumpsty, "Impellerdiffuser interaction in a centrifugal compressor," Journal of Turbomachinery, vol. 122, no. 4, pp. 777-786, 2000.

[11] K. Gallier, Experimental cHaracterization of High Speed Centrifugal Compressor Aerodynamic Forcing Function [Ph.D. thesis], Purdue University, West Lafayette, IN, USA, 2005.

[12] C. Robinson, M. Casey, B. Hutchinson, and R. Steed, "Impellerdiffuser interaction in centrifugal compressors," in Proceedings of the ASME Turbo Expo 2012: Turbine Technical Conference and Exposition, p. 767, Copenhagen, Denmark, 2012.

[13] R. T. Johnston and S. Fleeter, "Three-dimensional time-resolved inlet guide vane-rotor potential field interaction," Journal of Propulsion and Power, vol. 20, no. 1, pp. 171-179, 2004.

[14] R. T. Johnston and S. Fleeter, "Inlet guide vane wakes including rotor effects," Journal of Fluids and Structures, vol. 15, no. 2, pp. 235-253, 2001.

[15] T. F. McKain and G. J. Holbrook, Coordinates for a High Performance 4:1 Pressure Ratio Centrifugal Compressor, Indiana, Indianapolis, July 1997.

[16] "NUMECA International NUMECA FINE/Turbo User Manual 11.1," http://www.numeca.com. 


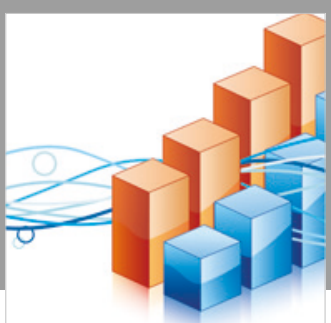

Advances in

Operations Research

\section{-n-m}
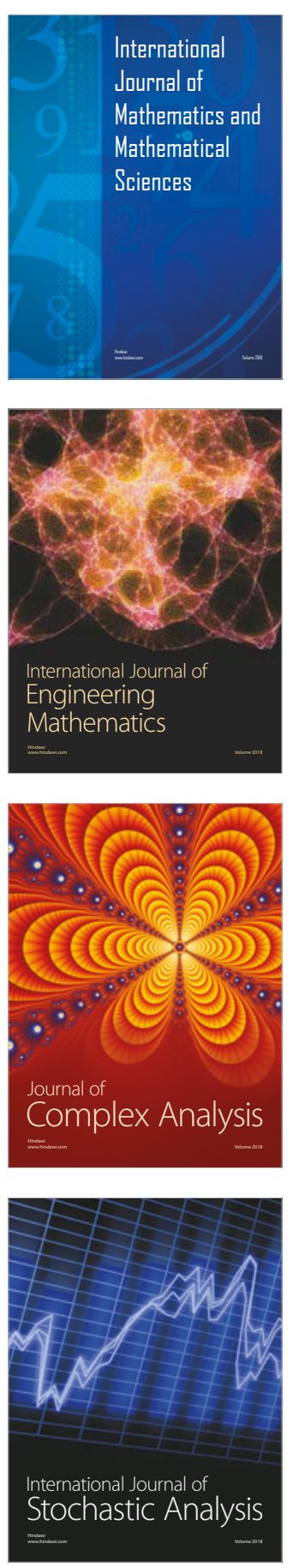
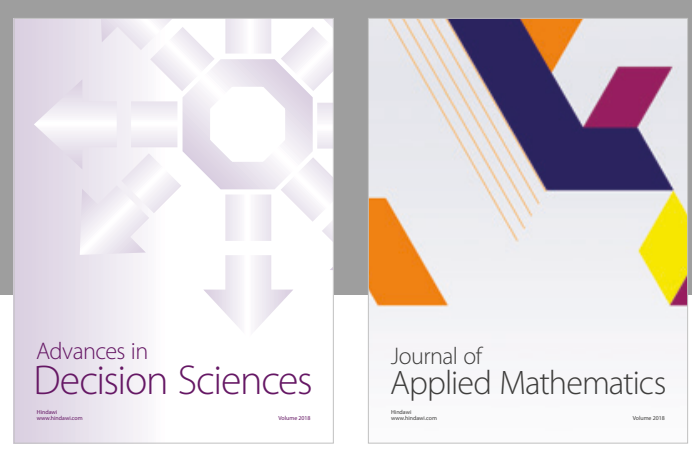

Journal of

Applied Mathematics
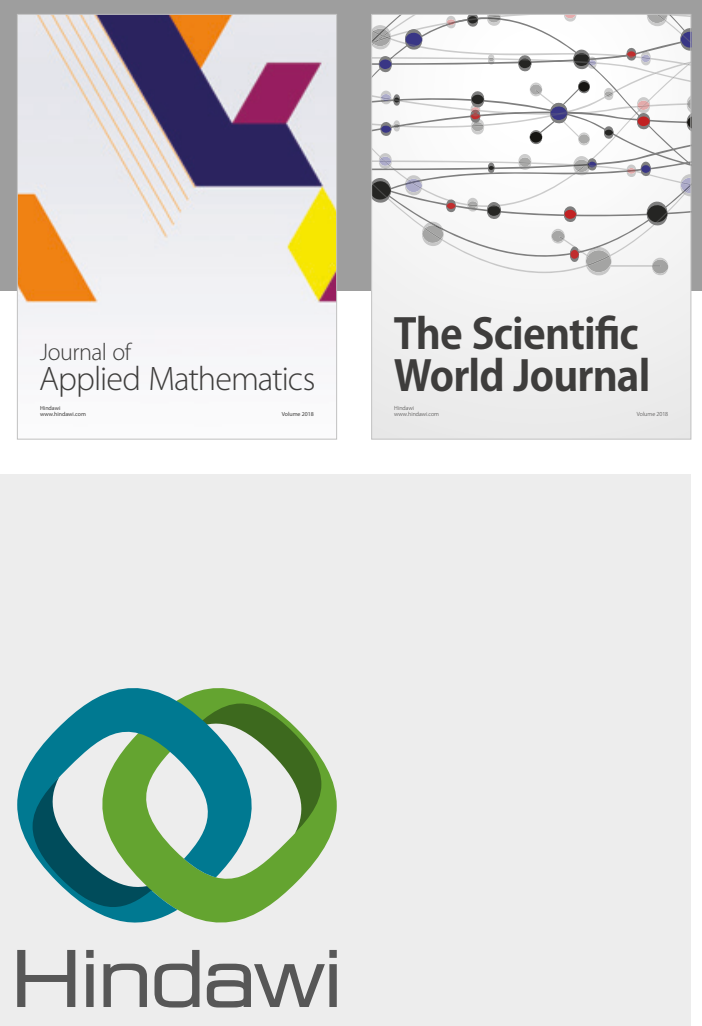

Submit your manuscripts at

www.hindawi.com

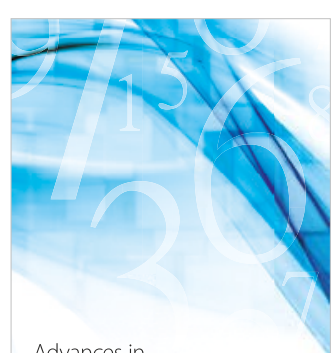

Advances in
Numerical Analysis
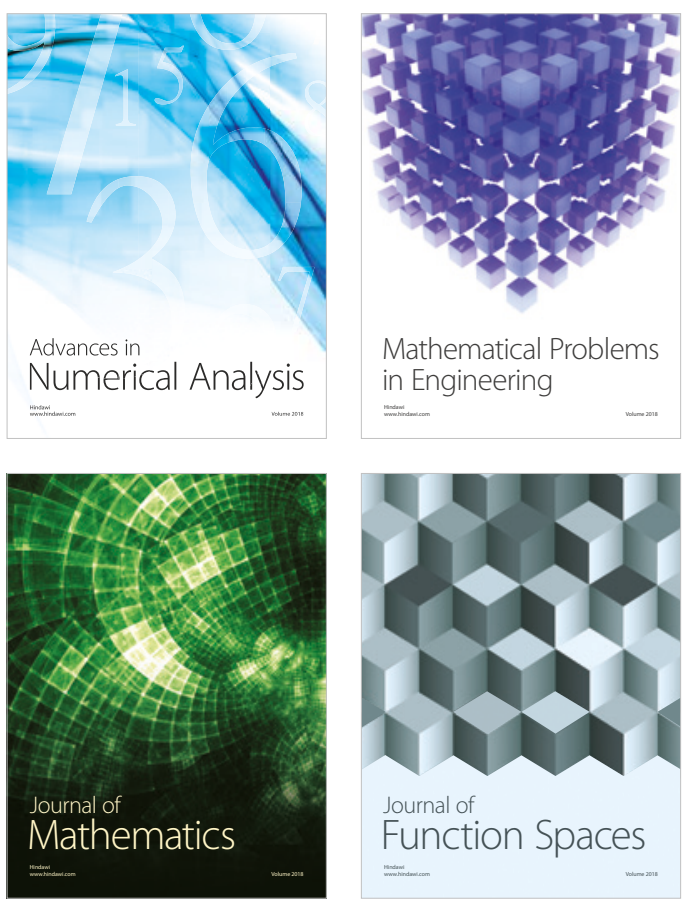

Mathematical Problems in Engineering

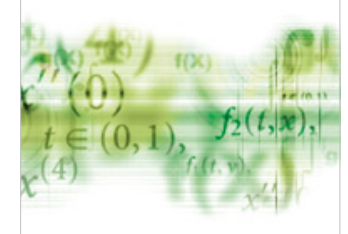

International Journal of

Differential Equations

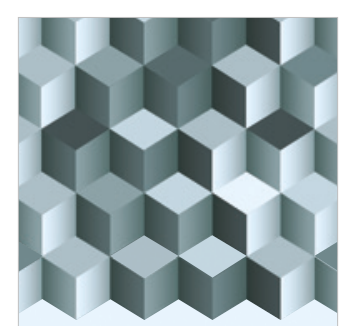

Journal of

Function Spaces

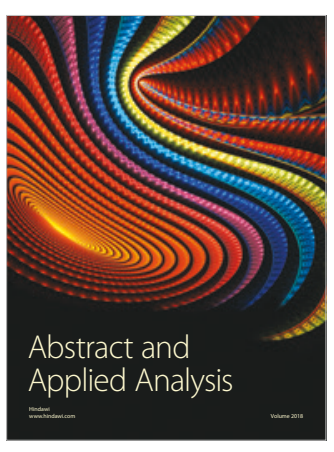

The Scientific

World Journal

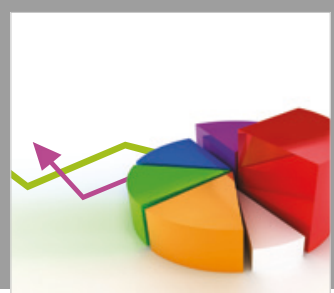

Journal of

Probability and Statistics
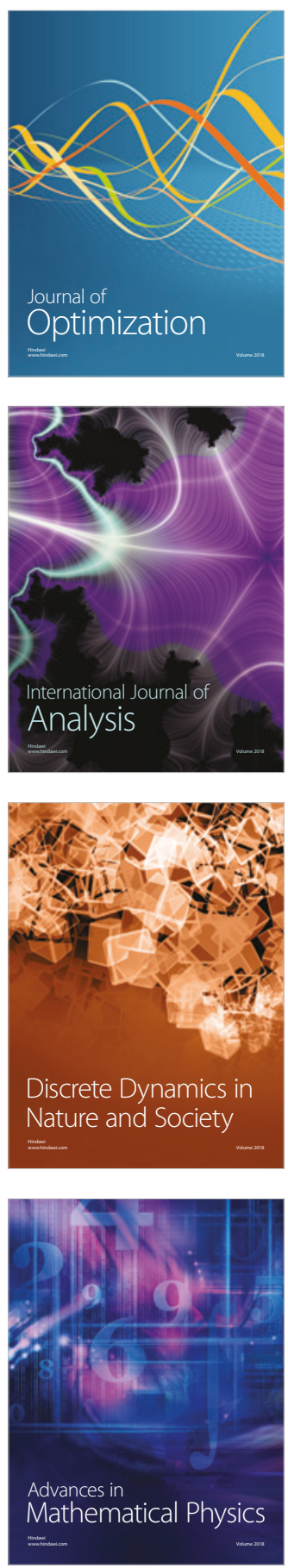